\title{
Continuity and anomalous fluctuations in random walks in dynamic random environments: numerics, phase diagrams and conjectures
}

\author{
Avena, L ; Thomann, P
}

\begin{abstract}
We perform simulations for one dimensional continuous-time random walks in two dynamic random environments with fast (independent spin-flips) and slow (simple symmetric exclusion) decay of space-time correlations, respectively. We focus on the asymptotic speeds and the scaling limits of such random walks. We observe different behaviors depending on the dynamics of the underlying random environment and the ratio between the jump rate of the random walk and the one of the environment. We compare our data with well known results for static random environment. We observe that the non-diffusive regime known so far only for the static case can occur in the dynamical setup too. Such anomalous fluctuations give rise to a new phase diagram. Further we discuss possible consequences for more general static and dynamic random environments
\end{abstract}

DOI: https://doi.org/10.1007/s10955-012-0502-1

Posted at the Zurich Open Repository and Archive, University of Zurich

ZORA URL: https://doi.org/10.5167/uzh-156513

Journal Article

Published Version

Originally published at:

Avena, L; Thomann, P (2012). Continuity and anomalous fluctuations in random walks in dynamic random environments: numerics, phase diagrams and conjectures. Journal of Statistical Physics, 147(6):10411067.

DOI: https://doi.org/10.1007/s10955-012-0502-1 


\title{
Continuity and Anomalous Fluctuations in Random Walks in Dynamic Random Environments: Numerics, Phase Diagrams and Conjectures
}

\author{
L. Avena $\cdot$ P. Thomann
}

Received: 16 January 2012 / Accepted: 18 May 2012 / Published online: 6 June 2012

(C) Springer Science+Business Media, LLC 2012

\begin{abstract}
We perform simulations for one dimensional continuous-time random walks in two dynamic random environments with fast (independent spin-flips) and slow (simple symmetric exclusion) decay of space-time correlations, respectively. We focus on the asymptotic speeds and the scaling limits of such random walks. We observe different behaviors depending on the dynamics of the underlying random environment and the ratio between the jump rate of the random walk and the one of the environment. We compare our data with well known results for static random environment. We observe that the non-diffusive regime known so far only for the static case can occur in the dynamical setup too. Such anomalous fluctuations give rise to a new phase diagram. Further we discuss possible consequences for more general static and dynamic random environments.
\end{abstract}

Keywords Random environments $\cdot$ Random walks $\cdot$ Law of large numbers $\cdot$ Scaling limits $\cdot$ Particle systems $\cdot$ Numerics

\section{Introduction}

1.1 Random Walk in Static and Dynamic Random Environments

Random Walks in Random Environments (RWRE) on the integer lattice are RWs on $\mathbb{Z}^{d}$ evolving according to random transition kernels, i.e., their transition probabilities/rates depend on a random field (static case) or a stochastic process (dynamic case) called Random Environment (RE). Such models play a central role in the field of disordered systems of particles. The idea is to model the motion of a particle in an inhomogeneous medium. In contrast with standard homogeneous RW, RWRE may show several unusual phenomena as non-ballistic transience, non-diffusive scalings, sub-exponential decay for large deviation probabilities. All these features are due to impurities in the medium that produce trapping

L. Avena $(\bowtie) \cdot$ P. Thomann Institut für Mathematik, Universität Zürich, Winterthurerstrasse 190, Zürich 8057, Switzerland e-mail: luca.avena@math.uzh.ch 
effects. Although they have been intensively studied by the physics and mathematics communities since the early 70's, except for the one-dimensional static case and few other particular situations, most of the results are of qualitative nature, and their behavior is far from being completely understood. We refer the reader to [23, 26] and [1, 14] for recent overviews of the state of the art in static and dynamic REs, respectively.

In this paper we focus on two one-dimensional models in dynamic RE. In particular, the RW will evolve in continuous time in (two-states) REs given by two well known interacting particle systems: independent spin flip and simple symmetric exclusion dynamics. Several classical questions regarding these types of dynamical models are still open while the behavior of the analogous RW in a i.i.d. static case is completely understood. We perform simulations focusing on their long term behavior. We see how such asymptotics are influenced as a function of the jump rate $\gamma$ of the dynamic REs. The idea is that by tuning the speed of the REs, we get close to the static RE ( $\gamma$ close to 0 ) or to the averaged medium ( $\gamma$ approaching infinity). We observe different surprising phases which allow us to set some new challenging conjectures and open problems.

Although the choice of the models could appear too restrictive and of limited interest, as it will be clear in the sequel, such particular examples present all the main features and the rich behavior of the general models usually considered in the RWRE literature. The conjectures we state can be extended to a more general setup (see Sect. 4.3).

The paper is organized as follows. In this section we define the models and give some motivation. In Sect. 2 we review the results known for the analogous RW in a static RE. Section 3 represents the main novel. We present therein the results of our simulations which shine a light on the behavior of the asymptotic speed (Sect. 3.1) and on the scaling limits of such processes (Sects. 3.2 and 3.3). When discussing each question we list several conjectures. In the last Sect. 4 we present a brief description of the algorithms, we discuss the robustness of our numerics and possible consequences for more general RE.

\subsection{The Model}

We consider a one-dimensional RW whose transition rates depend on a dynamic RE given by a particle system. In Sect. 1.2.1, we first give a rather general definition of particle systems and then we introduce the two explicit examples we will focus on. In Sect. 1.2.2 we define the RW in such dynamic REs.

\subsubsection{Random Environment: Particle Systems}

Let $\Omega=\{0,1\}^{\mathbb{Z}}$. Denote by $D_{\Omega}[0, \infty)$ the set of paths in $\Omega$ that are right-continuous and have left limits. Let $\left\{P^{\eta}, \eta \in \Omega\right\}$ be a collection of probability measures on $D_{\Omega}[0, \infty)$. A particle system

$$
\xi=\left(\xi_{t}\right)_{t \geq 0} \quad \text { with } \xi_{t}=\left\{\xi_{t}(x): x \in \mathbb{Z}\right\},
$$

is a Markov process on $\Omega$ with law $P^{\eta}$, when $\xi_{0}=\eta \in \Omega$ is the starting configuration. Given a probability measure $\mu$ on $\Omega$, we denote by $P^{\mu}(\cdot):=\int_{\Omega} P^{\eta}(\cdot) \mu(\mathrm{d} \eta)$ the law of $\xi$ when $\xi_{0}$ is drawn from $\mu$. We say that site $x$ is occupied by a particle (resp. vacant) at time $t$ when $\xi_{t}(x)=1$ (resp. 0).

Informally, a particle system is a collection of particles (1's) on the integer lattice evolving in a Markovian way. Depending on the specific transition rates between the different configurations, one obtains several types of particle systems. Each particle may interact with the others: the evolution of each particle is defined in terms of local transition rates 
that may depend on the state of the system in a neighborhood of the particle. For a formal construction, we refer the reader to Liggett [18], Chap. I.

In the sequel we focus on two well known examples with strong and weak mixing properties, respectively.

\section{(1) Independent Spin Flip (ISF)}

Let $\xi=\left(\xi_{t}\right)_{t \geq 0}$ be a one-dimensional independent spin-flip system, i.e., a Markov process on state space $\Omega$ with generator $L_{I S F}$ given by

$$
\left(L_{I S F} f\right)(\eta)=\sum_{x \in \mathbb{Z}}[\lambda \eta(x)+\gamma(1-\eta(x))]\left[f\left(\eta^{x}\right)-f(\eta)\right], \quad \eta \in \Omega,
$$

where $\lambda, \gamma \geq 0, f$ is any cylinder function on $\Omega, \eta^{x}$ is the configuration obtained from $\eta$ by flipping the state at site $x$.

In words, this process is an example of a non-interacting particle system on $\{0,1\}^{\mathbb{Z}}$ where the coordinates $\eta_{t}(x)$ are independent two-state Markov chains, namely, at each site (independently with respect to the other coordinates) particles flip into holes at rate $\lambda$ and holes into particles at rate $\gamma$. This particle system has a unique ergodic measure given by the Bernoulli product measure with density $\rho=\gamma /(\gamma+\lambda)$ which we denote by $v_{\rho}$ (see e.g. [18], Chap. IV).

\section{(2) Simple Symmetric Exclusion (SSE)}

The SSE is an interacting particle system $\xi$ in which particles perform a simple symmetric random walk at a certain rate $\gamma>0$ with the restriction that only jumps on vacant sites are allowed. Formally, its generator $L_{S S E}$, acting on cylinder functions $f$, is given by

$$
\left(L_{S S E} f\right)(\eta)=\gamma \sum_{\substack{x, y \in \mathbb{Z} \\ x \sim y}}\left[f\left(\eta^{x, y}\right)-f(\eta)\right], \quad \eta \in \Omega,
$$

where the sum runs over unordered neighboring pairs of sites in $\mathbb{Z}$, and $\eta^{x, y}$ is the configuration obtained from $\eta$ by interchanging the states at sites $x$ and $y$.

It is known (see [18], Chap. VIII) that the family of Bernoulli product measures $v_{\rho}$, with density $\rho \in(0,1)$, characterizes the set of equilibrium measures for this dynamics.

Remark 1.1 Note that the ISF and the SSE are completely different types of dynamics. They are both Markovian in time but while the ISF has no spatial correlations, the SSE has spacetime correlations. The ISF has very good mixing properties due to the fact that once $\gamma$ and $\lambda$ are given, no matter of the starting configuration, it will converge exponentially fast to the unique equilibrium given by $v_{\rho}$ with $\rho=\gamma /(\gamma+\lambda)$. On the contrary, the SSE dynamics is strongly dependent on the starting configuration and therefore does not satisfy any uniform mixing condition. In fact, it is a conservative type of dynamics with a family of equilibria given by $\left\{v_{\rho}: \rho \in(0,1)\right\}$. Because of these substantial differences, in the sequel we will informally say that the ISF and the SSE are examples of fast and slowly mixing dynamics, respectively.

\subsubsection{RW on Particle Systems}

Conditional on the particle system $\xi$, let

$$
X=\left(X_{t}\right)_{t \geq 0}
$$


be the continuous time random walk jumping at rate 1 with local transition probabilities

$$
\begin{array}{lll}
x \rightarrow x+1 & \text { at rate } & p \xi_{t}(x)+(1-p)\left[1-\xi_{t}(x)\right], \\
x \rightarrow x-1 & \text { at rate } & (1-p) \xi_{t}(x)+p\left[1-\xi_{t}(x)\right],
\end{array}
$$

with

$$
p \in[1 / 2,1) .
$$

In words, the RW $X$ jumps according to an exponential clock with rate 1 , if $X$ is on occupied sites (i.e. $\xi_{t}\left(X_{t}\right)=1$ ), it goes to the right with probability $p$ and to the left with probability $1-p$, while at vacant sites it does the opposite.

We write $P_{0}^{\xi}$ to denote the law of $X$ starting from $X(0)=0$ conditional on $\xi$, and

$$
\mathbb{P}_{\mu, 0}(\cdot)=\int_{D_{\Omega}[0, \infty)} P_{0}^{\xi}(\cdot) P^{\mu}(\mathrm{d} \xi)
$$

to denote the law of $X$ averaged over $\xi$. We refer to $P_{0}^{\xi}$ as the quenched law and to $\mathbb{P}_{\mu, 0}$ as the annealed law. In what follows, when needed, we will denote by

$$
X(p, \gamma, \rho),
$$

the RW $X$ just defined either in the ISF or in the SSE environment starting from $v_{\rho}$ and jumping at rate $\gamma$. Note that in the ISF case, the parameter $\lambda$ is uniquely determined once we fix $\gamma$ and $\rho$.

From now on we assume w.l.o.g. $\rho \in[1 / 2,1)$. The choice of $p, \rho \in[1 / 2,1)$ is not restrictive, indeed, due to symmetry, it is easy to see the following equalities in distribution

$$
X(p, \rho, \gamma) \stackrel{\mathbb{P}}{=} X(1-p, 1-\rho, \gamma) \stackrel{\mathbb{P}}{=}-X(p, 1-\rho, \gamma)
$$

\subsection{On Mixing Dynamics}

In our models, the REs at each site have only two possible states ( 0 or 1$)$, in most of the literature on RWRE, the models are defined in a more general framework where infinitely many states are allowed. The first paper dealing with RW in dynamic RE goes back to [9]. Since then, there has been intensive activity and several advances have recently been made showing mostly LLN, invariance principles and LDP under different assumptions on the REs or on the transition probabilities of the walker. See for example [3, 5, 7, 8, 10, 14, 19] (most of these references are in a discrete-time setting). For an extensive list of reference we refer the reader to $[1,14]$.

One of the main difficulties in the analysis of random media arises when space-time correlations in the RE are allowed. Both models presented in Sect. 1.2.1 fit in this class but, as mentioned in Remark 1.1, their mixing properties are substantially different. The ISF dynamics belongs to the class of fast mixing environments which is known to be qualitatively similar to a homogeneous environment. In fact, a RW on this type of RE exhibits always diffusive scaling.

The SSE is an example of what we called slowly mixing dynamics. For a RW driven by these latter types of dynamics, we are not aware of any results other than [2, 4, 12]. One of the main result of our simulations is that the RW in (1.4) on the SSE, similarly to the RW in a static RE (see Sect. 2.2), may exhibit non-diffusive behavior (see Sect. 3.2). This latter result 
is related to trapping phenomena (see Sects. 2.4 and 2.6) and suggests that, when considering non-uniform slowly mixing environments, the medium looks substantially different than a homogeneous one. This is confirmed by the rigorous annealed large deviation results in [2] (see the last paragraph in Sect. 2.3). Note that these results are due to the correlation structure of the RE but also depend on the following essential ingredients which produce some strong trapping effect: the one dimensional setting, the RW and the SSE being both nearest-neighbor, and the presence of local drifts to the right and to the left for the RW.

\subsection{Particle Systems as Random Environments}

The reader may wonder why we consider random environments given by particle systems. Particle systems represent a natural physical example of a two-state dynamical RE. Particle system theory has been intensively developed in the last thirty years and results from this theory can be used in the present context (see e.g. $[1-4,12,13,20]$ ). Several results proven for such particular models can be extended to more general settings. Finally, as shown in this paper, these dynamics are not too complex from an algorithmic point of view allowing to obtain good approximations for the asymptotics.

\section{Static Case and Trapping Phenomena: Review}

We present in this section well known results for the analogous model in an i.i.d. static medium. Consider a static random environment $\eta \in\{0,1\}^{\mathbb{Z}}$ with law $v_{\rho}$, the Bernoulli product measure with density $\rho \in[1 / 2,1)$. Given a realization of $\eta$, let $X=\left(X_{t}\right)_{t \geq 0}$ be the random walk with transition rates (compare with (1.5))

$$
\begin{array}{lll}
x \rightarrow x+1 & \text { at rate } & p \eta(x)+(1-p)[1-\eta(x)]=c^{+}(\eta), \\
x \rightarrow x-1 & \text { at rate } & (1-p) \eta(x)+p[1-\eta(x)]=c^{-}(\eta),
\end{array}
$$

with

$$
p \in[1 / 2,1)
$$

\subsection{Recurrence and LLN}

In [22] it is shown that $X$ is recurrent when $\rho=\frac{1}{2}$ and transient to the right when $\rho>\frac{1}{2}$. In the transient case both ballistic and non-ballistic behavior occur (see Fig. 1), namely, $\lim _{t \rightarrow \infty} X_{t} / t=v_{\text {static }}$ exists for $\mathbb{P}_{v_{\rho}}$-a.e. $\eta$, and

$$
v_{\text {static }} \begin{cases}=0 & \text { if } \rho \in\left[\frac{1}{2}, p\right], \\ >0 & \text { if } \rho \in(p, 1] .\end{cases}
$$

In particular, for $\rho \in(p, 1]$,

$$
v_{\text {static }}=v_{\text {static }}(\rho, p)=(2 p-1) \frac{\rho-p}{\rho(1-p)+p(1-\rho)}
$$


Fig. 1 The sides of this square represent degenerate cases. In particular when $p=1 / 2$ or $\rho=1$, the RW $X$ does not feel anymore the environment behaving as a Simple Symmetric Random Walk (SSRW in the picture) or as a homogeneous RW with drift $2 p-1$ (RW(p) in the picture), respectively. When $p=1$ we are in a trivial degenerate case. When $\rho=1 / 2$ we are in the recurrent case. Inside the square, by (2.3), above the diagonal we have transience with positive speed, while at and below the diagonal a non-ballistic transient regime holds, i.e. transience at zero-speed

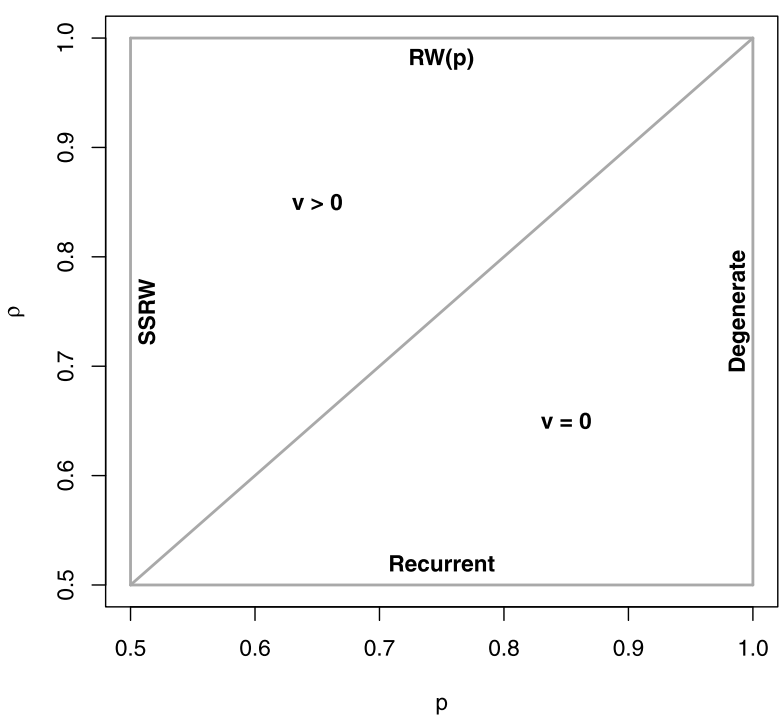

\subsection{Scaling Limits}

The scaling limits of one-dimensional RWRE have been derived in quite a general framework in a series of papers (see $[17,21]$ and $[25,26]$ for a review of those results and references). It turns out that diffusive, super-diffusive or sub-diffusive regimes can occur. By diffusive regime, we mean that $X_{t}-v t$ divided by $\sqrt{t}$ converges in distribution to a nondegenerate Gaussian distribution, while we refer to super- or sub-diffusive regime when $X_{t}-v t$ has to be rescaled by some factor of order $t^{\alpha}$, with $\alpha$ bigger or smaller than $1 / 2$, respectively, to converge weakly to some non-degenerate distribution. We now review the different scalings in the i.i.d. static RE. In what follows we focus only on the annealed law.

When $X$ is recurrent, [21] showed that $X$ is extremely sub-diffusive and it converges weakly to a non-degenerate random variable $Z$, namely,

$$
\frac{\sigma^{2} X_{t}}{(\log t)^{2}} \underset{t \rightarrow \infty}{\stackrel{\left(\mathbb{P}_{v_{\rho}}\right)}{\longrightarrow}} Z,
$$

where $\sigma^{2}$ is a positive constant and $Z$ is a random variable with a non-trivial law that was later identified by Kesten [16]. In this case, $X$ is called Sinai's random walk.

When $X$ is right-transient, [17] proved that the key quantity to determine the right scaling is the root $s$ of the equation

$$
\mathbb{E}_{v_{\rho}}\left[\left(\frac{c^{-}(\eta)}{c^{+}(\eta)}\right)^{s}\right]=1
$$

where $c^{-}(\eta)$ and $c^{+}(\eta)$ represent the rates to jump to the left and to the right, respectively (see (2.1)). In particular, they proved that when $s>2, X$ is diffusive with Gaussian limiting distribution, while for $s \in(0,2]$ super- or sub-diffusivity occur with some non-trivial stable law of parameters $(s, b)$ as limiting distribution ( $b$ is a constant, see also Theorem 2.3 in [26] for more details and references). The proof is based on the analysis of hitting times and makes use of the extra assumption that $\log \left[\frac{c^{-}(\eta)}{c^{+}(\eta)}\right]$ has a non-arithmetic distribution. This 
Fig. 2 The sides of the square represent degenerate cases (see Fig. 1). When $\rho=1 / 2$ we are in the so called Sinai case in which $X$ is recurrent and extremely sub-diffusive. We call "leaf" the region around the diagonal delimited by the curves

$f_{1}(p)=(1-p)^{2} / p^{2}$ and

$f_{2}(p)=(p-\sqrt{p(1-p)}) /$

$(2 p-1)$ corresponding to $s=2$ and $s=0.5$, respectively. The area above the leaf corresponds to the diffusive case with $s>2$, inside the leaf we have the super-diffusive regime while in the lower remaining region for $s \in(0,0.5)$ we have the sub-diffusive regime

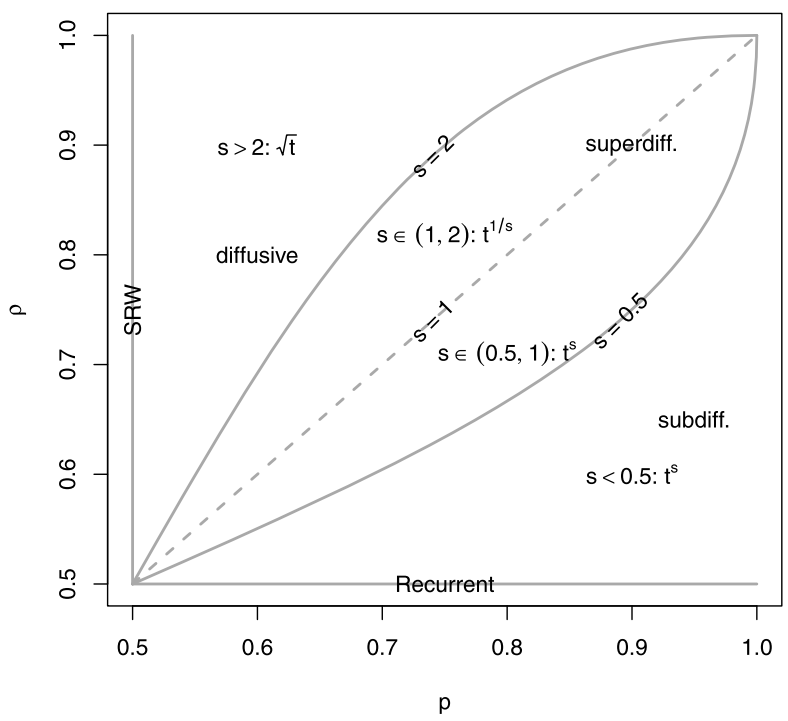

latter is a delicate technical assumption (see also Remark 3 in [17]) not satisfied in our model since

$$
\log \left[\frac{c^{-}(\eta)}{c^{+}(\eta)}\right]=[2 \eta(0)-1] \log \left(\frac{1-p}{p}\right)
$$

does have an arithmetic distribution. At the present state of the art, the role of this assumption is not entirely clear. There are examples in the literature in which by dropping it, the convergence in distribution does not hold (see e.g. [6] Sect. 8 and references therein). For our model (2.1), we performed simulations (see Figs. 3 and 4) which clearly suggest that the arithmetic law of (2.7) does not play any role, namely, the scaling behavior of $X$ is like in the general case under the assumption of a non-arithmetic law. The following list summarizes the different scalings of $X$.

- Diffusive: $s>2$, scaling order $\sqrt{t}$.

- Super-diffusive: $s \in(0.5,2)$.

$-s \in(1,2)$ : scaling order $t^{1 / s}$.

$-s=1$ : scaling order $t / \log t$.

- $s \in(0,1)$ : scaling order $t^{s}$.

- Diffusive: $s=0.5$, scaling order $\sqrt{t}$.

- Sub-diffusive: $s \in(0,0.5)$, scaling order $t^{s}$.

In our model, the explicit solution of (2.6) is given by

$$
s=s(p, \rho)=\frac{\log \left(\frac{1-\rho}{\rho}\right)}{\log \left(\frac{1-p}{p}\right)}>0, \quad \text { for } p, \rho>1 / 2 .
$$

Figure 2 shows the phase diagram in $(\rho, p)$ of the regimes just described.

As we mentioned, we tested numerically the results presented so far (see Sect. 4.1 for a description of the algorithms we implemented). Figures 3 and 4 show that our numerics match the theoretical picture just described. 
Fig. 3 For each of the points marked with a black square, a dot or a cross, we performed numerical experiments (similar to those explained in Sect. 3.3) to determine the scaling exponents of $X=X(p, \rho)$. The symbols square, dot or cross mean that for the corresponding points, our numerical estimates gave a sub-, a super- or a diffusive scaling exponent, respectively. Note that except for a small region in between super-diffusive and diffusive regimes (it is reasonable to have numerical fluctuations close to a phase transition), the experiments confirm the theoretical picture. Figure 4 provides a few explicit examples of our numerics in the different regimes

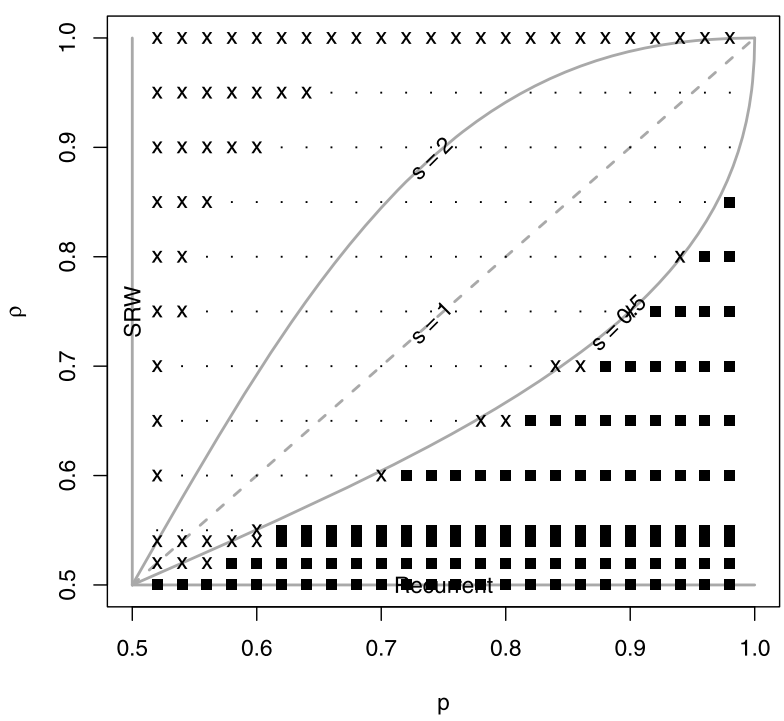

\subsection{Large Deviations for the Empirical Speed}

For the empirical speed of a one-dimensional RW in a static RE, quenched and annealed Large Deviation Principles (LDPs) and refined large deviations estimates have been obtained in a series of papers (see e.g. $[11,15,24,26]$ ). We just mention that for the RW $X$ defined through (2.1), when $v>0$, the rate function associated to the LDP at rate $t$ is zero on the whole interval $[0, v]$. Roughly speaking, this is saying that for $\theta \in[0, v), P\left(X_{t} / t \approx \theta\right)$ decays sub-exponentially in $t$. We recall that for homogeneous RW such a decay is always exponential. This phenomenon is due to trapping effects which we briefly introduce in the next section.

Large deviation estimates for the dynamical models in Sect. 1.2 were obtained in [2]. In particular, it is shown that in the ISF case the rate function has a unique zero (as for homogeneous RW) while in the SSE case, the rate function (at least under the annealed measure) presents a flat piece as we just described for the i.i.d. static case.

\subsection{Trapping Phenomena}

The anomalous behaviors like the transient regime at zero-speed, the non-diffusivity, as well as the sub-exponential decay of the large deviations probabilities we reviewed, are due to the presence of traps in the medium, i.e., localized regions where the walk spends a long time with a high probability. To get an intuition, Fig. 5 below shows an explicit example of a trap. For a deeper insight of trapping phenomena we should introduce the random potential representation of the environment for which we refer the reader to the literature (see e.g. [21] and other references in [26]). 
(1) Densities at $s=17.32$

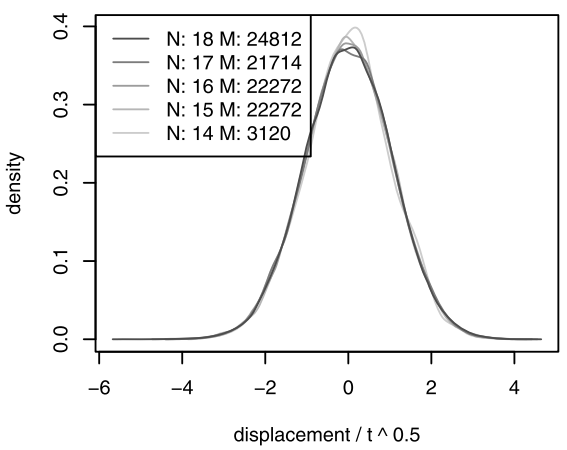

(3) Densities at $\mathrm{s}=\mathbf{0 . 7 6}$

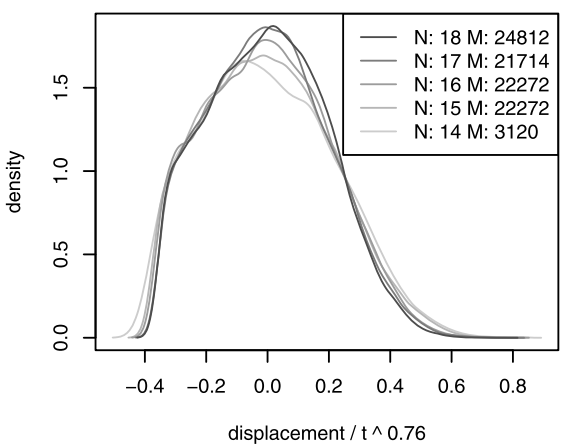

(5) Densities at $s=0$

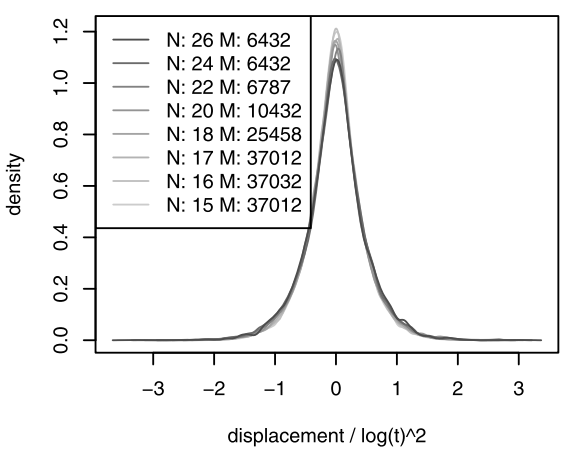

(2) Densities at $s=1.64$

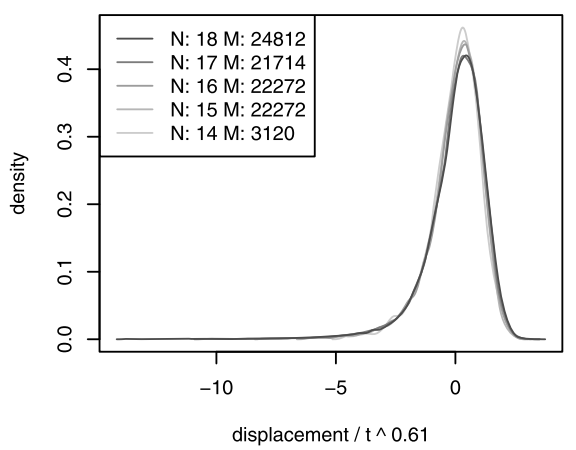

(4) Densities at $\mathbf{s}=\mathbf{0 . 3 6}$

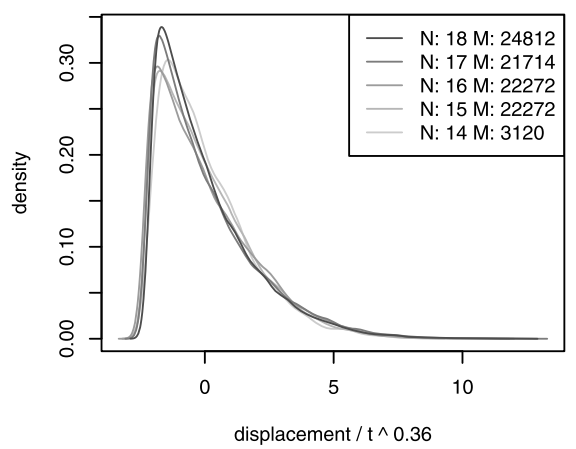

Phase diagram, scaling limits; static case

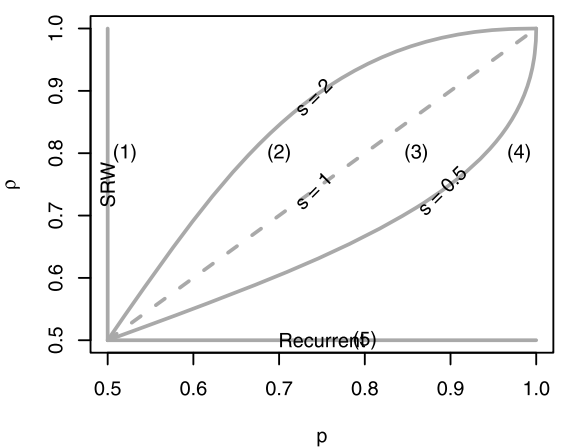

Fig. 4 A few explicit examples of the densities obtained via our numerics in each of the different scaling regimes. In the right bottom picture the $(p, \rho)$-points associated to each labeled plot are specified. In each plot we overlapped the densities obtained with independent experiments at the different times $n=2^{N}$, over samples of size $M$, properly rescaled. In particular, (1), (2), (3), (4) and (5) correspond to ballistic diffusive, ballistic super-diffusive, transient zero-speed super-diffusive, transient zero-speed sub-diffusive and recurrent Sinai case, respectively 


\section{$\longrightarrow+4 \rightarrow$

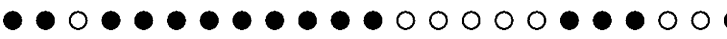

Fig. 5 An example of a trap, even though the global drift is to the right, i.e. $\rho>1 / 2$, a long interval with vacant sites creates a region with local drift against the global one. To cross such a trap $X$ needs an average number of trials that is exponential in the size of the interval

\subsection{The Averaged Medium}

In the sequel we will refer to the RW in the averaged medium, $Y$ (averaged), for the homogeneous nearest neighbor RW with transition rates

$$
\begin{array}{lll}
x \rightarrow x+1 & \text { at rate } & p \rho+(1-p)(1-\rho), \\
x \rightarrow x-1 & \text { at rate } & (1-p) \rho+p(1-\rho),
\end{array}
$$

where $\rho, p \in[1 / 2,1)$. It easily follows that such a RW is right transient as soon as $p>1 / 2$ and $\rho \neq 1 / 2$. Moreover, by the law of large numbers for i.i.d. sequences, we get that

$$
\begin{aligned}
\lim _{t \rightarrow \infty} \frac{\left.Y_{t} \text { (averaged }\right)}{t} & =v_{\text {averaged }}(p, \rho) \\
& =(2 \rho-1)(2 p-1), \quad \text { a.s. }
\end{aligned}
$$

This RW would correspond to the walker defined in (1.4) which observes at each lattice position a constant density of particles $\rho$. That is, between two jumps of the walker the environment is replaced by one that is an independent sample drawn from $v_{\rho}$.

In the dynamical models of Sect. 1.2.1, both particle systems are assumed to be in equilibrium with density $\rho$ and exhibit some decay of correlations in time. Hence, roughly speaking, if $\gamma$ approaches infinity, the environment becomes asymptotically independent between two jumps of the walker. Therefore, under the annealed law, we expect for both models that there is some convergence to the averaged medium as $\gamma \rightarrow \infty$.

\subsection{Towards the Dynamic RE: Dissolvence of Traps}

In the previous sections we saw that the RW $X$ in the static RE $\eta \in\{0,1\}^{\mathbb{Z}}$ sampled from the Bernoulli product measure $v_{\rho}$ presents "slow-down phenomena" due to the presence of traps. The dynamical models in Sect. 1.2.2 can be interpreted as the model in the static RE when we "switch on" some stochastic dynamics which allows particles of the RE to move (SSE dynamics) or to be created/annihilated (ISF dynamics). The natural question is then:

How does the dynamics of the random environment influence

the trapping effects present in the static case?

In the sequel we will present the outcome of simulations for the empirical speed of $X$ in the different REs of Sect. 1.2.1 and we will compare them with the static and the averaged medium case. Note that $v_{\rho}$ is an equilibrium measure for both particle systems we use: ISF and SSE.

At a heuristic level, one should expect that the evolution of particles in dynamic REs favors the dissolvence of traps, consequently the RW $X$ in the dynamic RE should be "faster" than in the static medium. In other words, the long stretches of holes present at time zero, and 

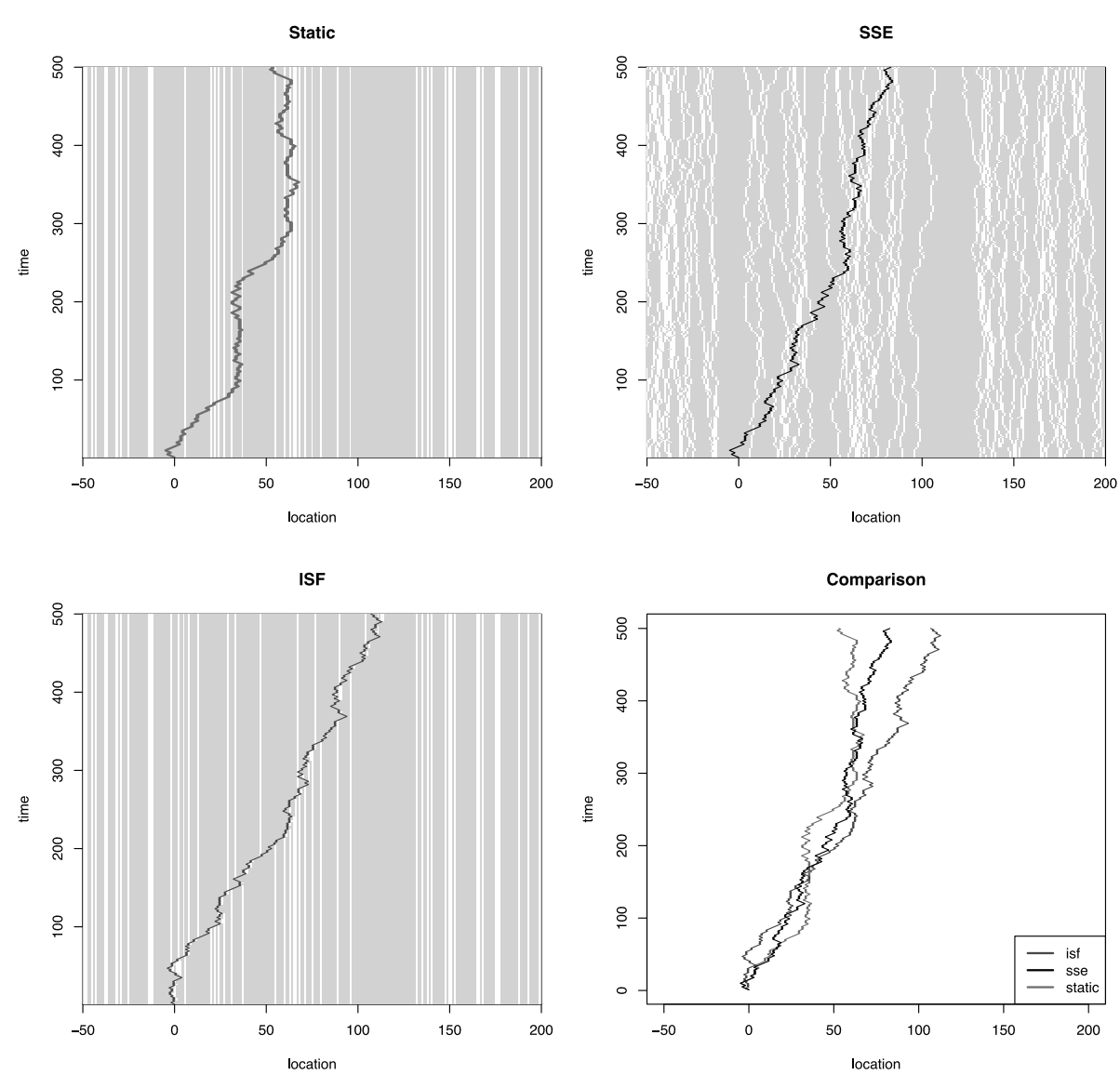

Fig. 6 Three simulated trajectories of $X$ in the static, ISF and SSE environments starting from the same configuration. $(p, \rho)=(0.7,0.8)$, and $\gamma=0.1$ for ISF and SSE. These trajectories are compared in the bottom right picture showing the intuitive domination mentioned above. The background of the other plots displays realizations of the corresponding RE. In particular gray and white mean presence or absence of particles, respectively. Note that in the ISF case, due to the independence in space of the dynamics, the RE has been updated only around the RW trajectory (see the description of the algorithm in Sect. 4.1)

responsible of the slow-down phenomena in the static case, get destroyed by the movement of particles in the dynamic case. As a counter effect the dynamics can create new traps during the evolution of the RW $X$. Nevertheless, in the static case, the traps are frozen, while in the dynamic case, all the traps have an a.s. finite survival time. Such intuitive arguments suggest that the displacement of the RW $X$ should be bigger in the dynamic case than in the static one. Figure 6 illustrates this intuitive domination; it represents simulated trajectories of the RW in the three different random environments (static, ISF, SSE) starting from the same configuration sampled from $v_{\rho}$ at a given $p$.

Furthermore, depending on the specific dynamics of the underlying particle system, the survival time and the nature of a typical trap have to be different. In fact, if we consider a trap as in Fig. 5 formed by an interval filled of holes. It is clear that in the ISF case, since particles can be created at each site at a given rate, such a trap gets easily destroyed. In the 
SSE cases, due to the conservation law, to dissolve such a trap, we have to wait for particles from outside the interval to invade it.

\section{Results and Conjectures}

In this section we finally present the outcome of the simulations for the asymptotics of $X$ in the different cases. Section 3.1 concerns the asymptotic speed as a function of the parameters $(\rho, p, \gamma)$ while Sects. 3.2 and 3.3 focus on the scaling limits. We give formal conjectures and discuss them based on the analysis of the data. The data will be presented in the form of figures. In the sequel, the jump rate $\gamma$ of the RE plays a central role. Throughout the paper we assumed, for simplicity, the RW jumping at rate 1, if instead we let it jump at any other rate $\lambda>0$, the same results would hold replacing $\gamma$ by $\gamma / \lambda$.

\subsection{Asymptotic Speed}

Denote by $X_{n}=X_{n}(\rho, p, \gamma)$ the position of the RW in (1.4) after $n$ exponential times of rate 1 . In this section we analyze the behavior of the asymptotic speed which we obtained by evaluating for large $n$

$$
v_{n}=v_{n}(\rho, p, \gamma):=\frac{1}{n M} \sum_{i=1}^{M} X_{n}^{(i)} \text {, }
$$

over a sample of $M$ independent experiments (the values of $M$ 's and $n$ 's will be specified in the figures.)

Conjecture 3.1 Let $\gamma>0,(p, \rho) \in[1 / 2,1) \times[1 / 2,1)$, and assume $\xi$ is the SSE, then $P^{\mu}-\xi$ a.s.

$$
\exists \lim _{t \rightarrow \infty} \frac{X_{t}(p, \rho, \gamma)}{t}=: v(p, \rho, \gamma) \in \mathbb{R}
$$

Conjecture 3.1 should hold in great generality at least for translation invariant RE. At the present state of the art, the existence of an almost sure constant speed has been proven for dynamic REs with "good" mixing properties in space and time (see $[3,5,13,20]$ ) except in [4] which instead uses a strong elliptic condition. In particular, Conjecture 3.1 is a rigorous statement if $\xi$ is the ISF (see $[3,20]$ ).

Conjecture 3.2 Let $\xi$ be either the SSE or the ISF. For any $\gamma>0, p \in(1 / 2,1)$, the function $\rho \longmapsto v(p, \rho, \gamma)$ is continuous and non-decreasing.

Note that the monotonicity is trivial once the existence of $v(p, \rho, \gamma)$ is given. Indeed, it follows by the fact that for any $\rho<\rho^{\prime}$, the RWs $X_{t}(p, \rho, \gamma)$ and $X_{t}\left(p, \rho^{\prime}, \gamma\right)$ can be coupled so that they remain ordered. Figure 7 below illustrates the mentioned monotonicity. In particular, it refers to the outcome of the numerics produced in the case of the SSE with $p=0.8$. We remark that the same qualitative picture holds in the ISF case and for any other choice of $p \in(1 / 2,1)$. 
Fig. 7 The lower and the upper dashed curves correspond to the speeds in the static (2.4) and the averaged medium (2.10) cases, respectively. The different solid curves represent the function $\rho \longmapsto v(0.8, \rho, \gamma)$ at the different $\gamma$ 's specified. In particular, each curve is obtained after interpolating 11 points at distance 0.05 , where each point has been obtained by averaging over a sample with at least $M=O\left(10^{3}\right)$ simulations of the empirical speed $v_{n}$ in (3.1) with $n=2^{16}$. This picture has been produced by using the SSE as RE

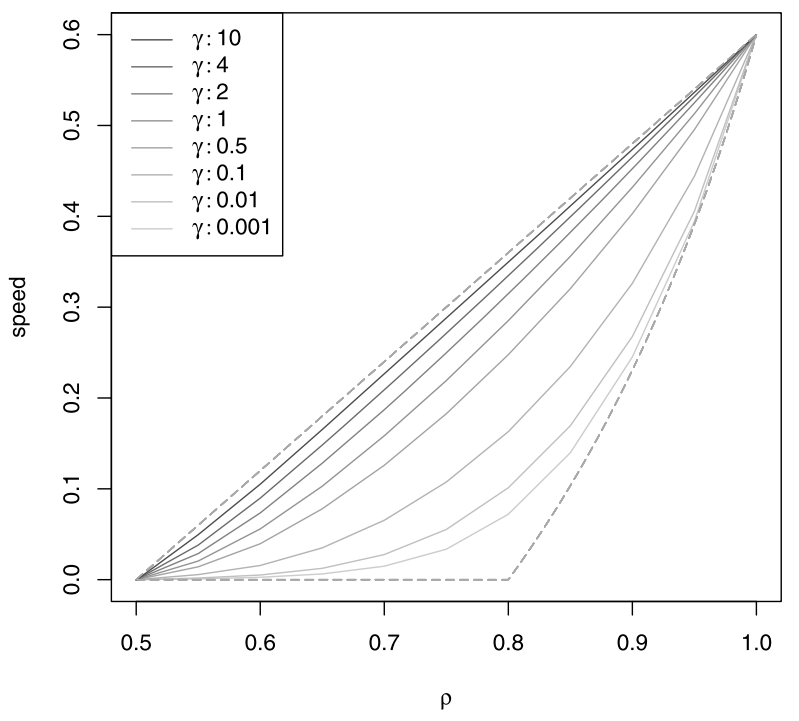

Conjecture 3.3 Let $\xi$ be either the SSE or the ISF. Then the function $\gamma \longmapsto v(p, \rho, \gamma)$ is continuous and non-decreasing. Moreover

$$
\begin{gathered}
\lim _{\gamma \downarrow 0} v(p, \rho, \gamma)=v_{\text {static }}(p, \rho), \\
\lim _{\gamma \rightarrow \infty} v(p, \rho, \gamma)=v_{\text {averaged }}(p, \rho) .
\end{gathered}
$$

Conjecture 3.3 is supported by the results of the experiments presented in Figs. 7 and 8. To state the next conjecture which describes the behavior of the speed as a function of $p$, we introduce some quantities defined in terms of $\gamma$ for fixed $\rho \in(1 / 2,1)$.

Conjecture 3.4 Let $\xi$ be either the SSE or the ISF. Fix $\rho \in(1 / 2,1)$. Define

$$
\begin{gathered}
\gamma_{1}(\rho):=\inf \{\gamma>0: v(p, \rho, \gamma)>0 \text { for all } p>1 / 2\}, \\
\gamma_{2}(\rho):=\inf \{\gamma>0: p \longmapsto v(p, \rho, \gamma) \text { is concave }\}, \\
\gamma_{3}(\rho):=\inf \{\gamma>0: p \longmapsto v(p, \rho, \gamma) \text { is non-decreasing }\} .
\end{gathered}
$$

Then, the function $p \longmapsto v(p, \rho, \gamma)$ is continuous. Moreover,

$$
0 \leq \gamma_{1}(\rho)<\gamma_{2}(\rho)<\gamma_{3}(\rho)<\infty \text {. }
$$

Conjecture 3.4 states the existence of several critical $\gamma$ 's for which we see a different behavior of the speed as a function of $p$. Figure 8 below shows such a scenario in the SSE case at a given $\rho=0.8$. Again, the same qualitative picture holds for any other $\rho \in(1 / 2,1)$ or by considering the ISF. Above $\gamma_{3}(\rho)$ the function $p \longmapsto v(p, \rho, \gamma)$ is increasing, while it starts to become non-monotone for $\gamma<\gamma_{3}(\rho)$. Below $\gamma_{2}(\rho)$ it looses the concavity and 
Fig. $8 \rho=0.8$. The lower and the upper dashed curves correspond to the speeds in the static (2.4) and the averaged medium (2.10) cases, respectively. The different solid curves represent the function $p \longmapsto v(p, 0.8, \gamma)$ at the different specified $\gamma$ 's. Each solid curve is obtained after interpolating 26 points at distance 0.02 , where each point has been obtained by averaging over the outcome of samples with at least $M=O\left(10^{3}\right)$ independent simulations of the empirical speed $v_{n}$ in (3.1) with $n \geq 2^{18}$. This plot has been produced by using the SSE as a RE

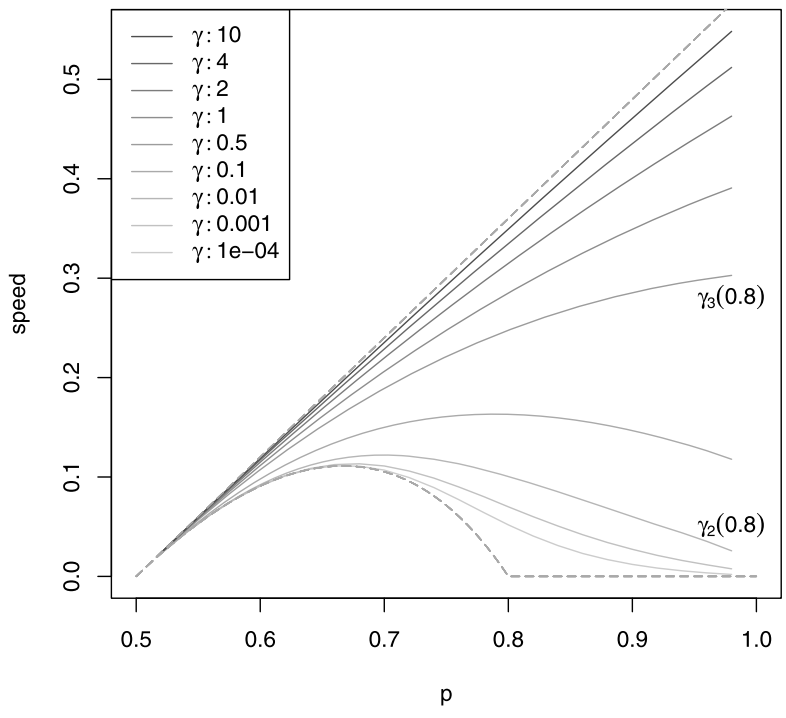

for $\gamma \leq \gamma_{1}(\rho)$ it possibly starts to have a vanishing piece. A crucial issue is to understand whether

$$
\gamma_{1}(\rho)>0 .
$$

The positivity of $\gamma_{1}(\rho)$ would imply a surprising transient regime with zero speed which so far has been proven only in the static case (see Sect. 2.1). In the case of the ISF, it follows from [20] that $\gamma_{1}(\rho)=0$. It might still be that $\gamma_{1}(\rho)>0$ in the SSE case. Unfortunately, we feel not able to conjecture anything based on our numerics since on one hand in the corresponding region $\gamma \ll 1$, at the time scales we could achieve, the simulation may just be a weak perturbation of the static case, and on the other hand this is a very delicate phenomenon to test statistically since of course in any transient regime the expected speed at finite time is strictly positive. We therefore leave (3.8) as a key open problem of this model.

The loss of monotonicity for low $\gamma$ 's is related to the strength of the traps. In the static case, the speed $p \longmapsto v(p, \rho, \gamma)$ looks like the dashed lower curve in Fig. 8 at any fixed $\rho \in(1 / 2,1)$. For $p$ big enough it starts to become decreasing until it vanishes. Intuitively, this is saying that when we increase $p$, no matter what the size of a typical trap is, the holes tend to act almost as reflecting barriers.

Figure 9 presents a quantitative version of Conjecture 3.4 in both the ISF and SSE cases.

\subsection{Scaling Limits and Phase Diagram: SSE}

We now turn to the analysis of the scaling limit of $X$. The ISF case belongs to the class of dynamic RE with strong mixing properties and it is known that for such dynamics for any $\gamma>0, X$ satisfies a functional CLT (see e.g. [20]). In the sequel we therefore focus only on the SSE case. We just mention that we checked numerically the known diffusivity of the ISF case. We obtained excellent agreement in this case except for very few points close to degenerate cases, i.e. for $\gamma$ too small and $(p, \rho) \approx(1,1)$ (see also the discussion in Sect. 4.2).

Our main conjecture is: 
Fig. 9 Quantitative phase diagrams describing Conjecture 3.4 in the ISF and the SSE environments. To each $(\rho, \gamma)$ we associate the symbols $m, c$ or + . They mean that the corresponding speed is a monotone, concave and non-concave function of $p \cdot \gamma_{3}(\rho)$ would correspond to the curve in between the regions of $m$ 's and $c$ 's, while $\gamma_{2}(\rho)$ would be the line separating the regions with $c$ 's and +'s

\section{Critical gamma: isf}

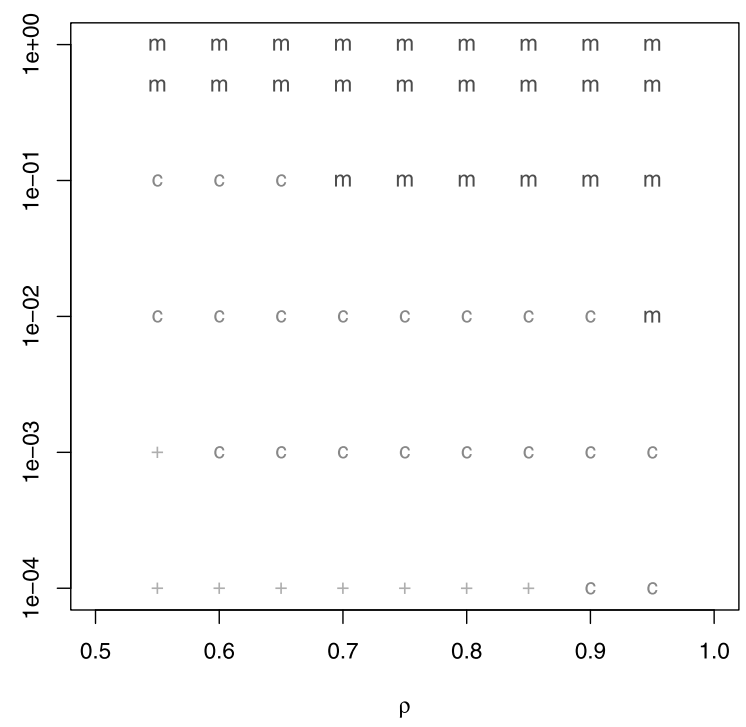

Critical gamma: sse

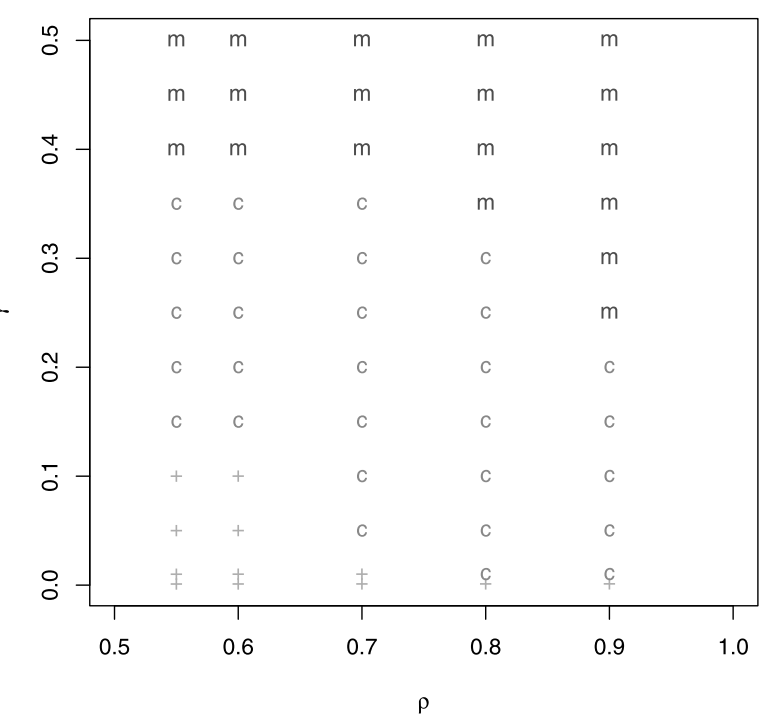

Conjecture 3.5 Let $\xi$ be the SSE. There exist two monotone functions $\widetilde{\gamma_{1}}, \widetilde{\gamma_{2}}:[1 / 2,1)^{2} \longmapsto$ $\mathbb{R}^{+}$with

(i) $\tilde{\gamma}_{i}$ equals zero only on the sets $A_{i}, i=1,2$, defined by

$$
A_{1}:=\left\{(p, \rho) \in[1 / 2,1]^{2}: \rho \leq f_{1}(p)\right\}
$$


Fig. 10 A qualitative picture of the phase diagram described in Conjecture 3.5 in the degenerate cases $\rho=1 / 2$ (recurrent case) and $p=1 / 2$ (SSRW). The lower and upper dotted curves at $\rho=1 / 2$ represent $\tilde{\gamma_{1}}(p, 1 / 2)$ and $\tilde{\gamma_{2}}(p, 1 / 2)$, respectively

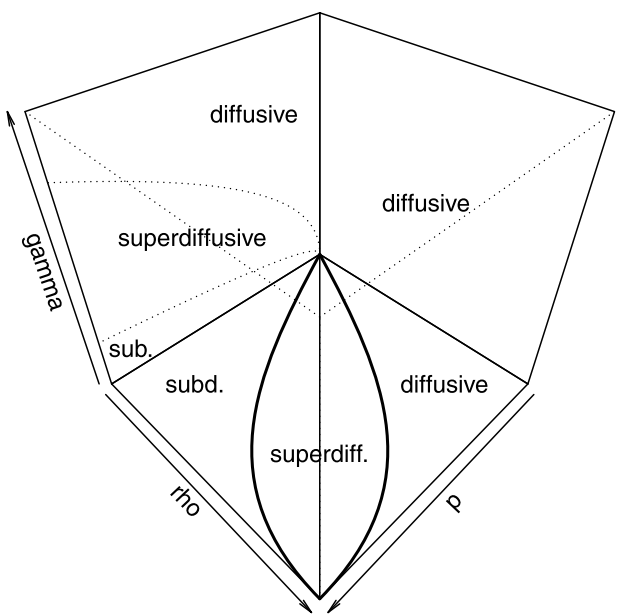

$$
A_{2}:=\left\{(p, \rho) \in[1 / 2,1]^{2}: \rho \leq f_{2}(p)\right\},
$$

with $f_{1}(p)=\frac{(1-p)^{2}}{p^{2}}$ and $f_{2}(p)=\frac{p-\sqrt{p(1-p)}}{2 p-1}$ (see Fig. 2),

(ii) ${\tilde{\gamma_{1}}}_{\gamma_{2}}$ whenever they are non-zero,

(iii) $\tilde{\gamma}_{i}$ is increasing in $p$ and decreasing in $\rho$, for any $(p, \rho) \in[1 / 2,1)^{2} \backslash A_{i}, i=1,2$,

and such that for any $(p, \rho) \in[1 / 2,1)^{2}$, we have the following cases

1. $X(p, \rho, \gamma)$ is sub-diffusive for any $\gamma<\tilde{\gamma_{1}}(p, \rho)$

2. $X(p, \rho, \gamma)$ is super-diffusive for any $\tilde{\gamma_{1}}(p, \rho)<\gamma<\tilde{\gamma_{2}}(p, \rho)$

3. $X(p, \rho, \gamma)$ is diffusive for any $\gamma>\tilde{\gamma_{2}}(p, \rho)$ or at $\gamma=\tilde{\gamma_{1}}(p, \rho)$

This conjecture is the most interesting novel result of our numerics. Figures 10 and 11 show the qualitative scenario stated in Conjecture 3.5. Recall the "super-diffusive leaf" in Fig. 2, note that the functions $f_{1}$ and $f_{2}$ represent the lower and the upper boundary of the leaf, respectively. The fact that for the ISF (and more generally for RE with space-time correlation with exponential or fast polynomial decay) at any $\gamma>0$ we have a diffusive scaling, can be rephrased by saying that for any $\gamma>0$ the leaf vanishes and any point $(p, \rho) \in[1 / 2,1)^{2}$ corresponds to diffusive regime. On the other hand, in case of the SSE, Conjecture 3.5 says that as soon as we switch on the SSE dynamics (i.e. for small $\gamma>0$ ) the leaf is still present and starts to move towards the $p$-axis as $\gamma$ increases until a certain critical $\gamma$ for which the leaf completely disappears. Note that the disappearance of the leaf for $\gamma$ big enough is consistent with the fact that as $\gamma$ increases we get closer and closer to the averaged medium case which is clearly diffusive. This observed phenomenon (although it could still be local, see Sect. 4.2) suggests that due to the slow-mixing properties of the exclusion dynamics, traps play a crucial role to determine the scaling limit of $X$. In particular, depending on the ratio of the jump rate of the walker and the one of the SSE, we can observe diffusivity or not.

Figures 12, 13, 14, 15, 16 show some of the data supporting Conjecture 3.5. In Sect. 3.3 we describe how we obtained these phase diagrams. 

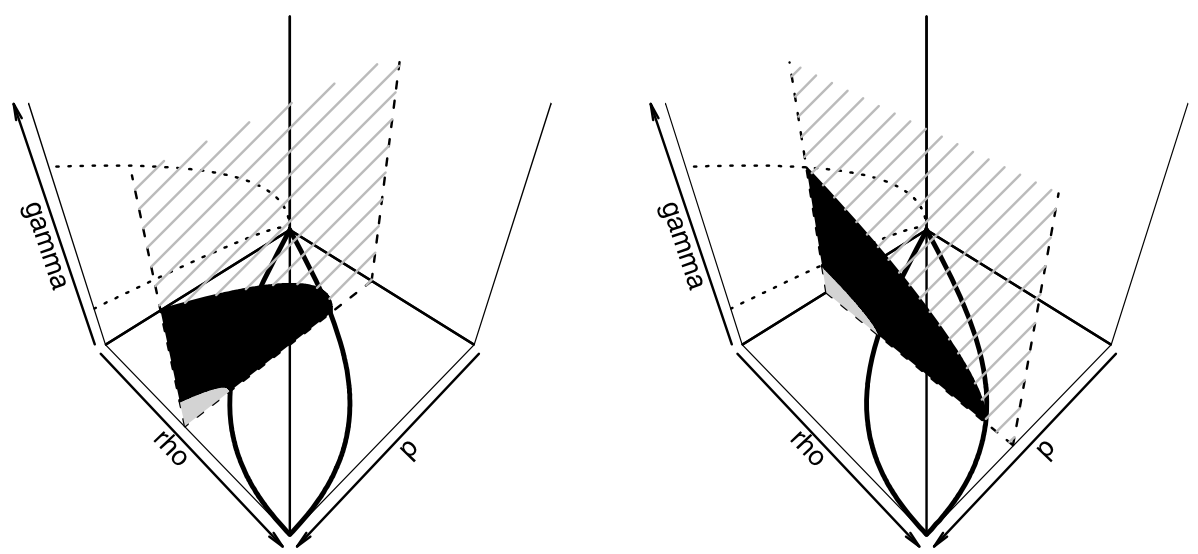

Fig. 11 Two qualitative sections of the phase diagram described in Conjecture 3.5 for fixed $\rho$ (on the left) and $p$ (on the right). The areas of those sections in gray, black and striped correspond to sub-diffusive, super-diffusive and diffusive regimes, respectively. In particular, the lower and upper curves bounding the black areas represent $\tilde{\gamma_{1}}$ and $\tilde{\gamma_{2}}$, respectively
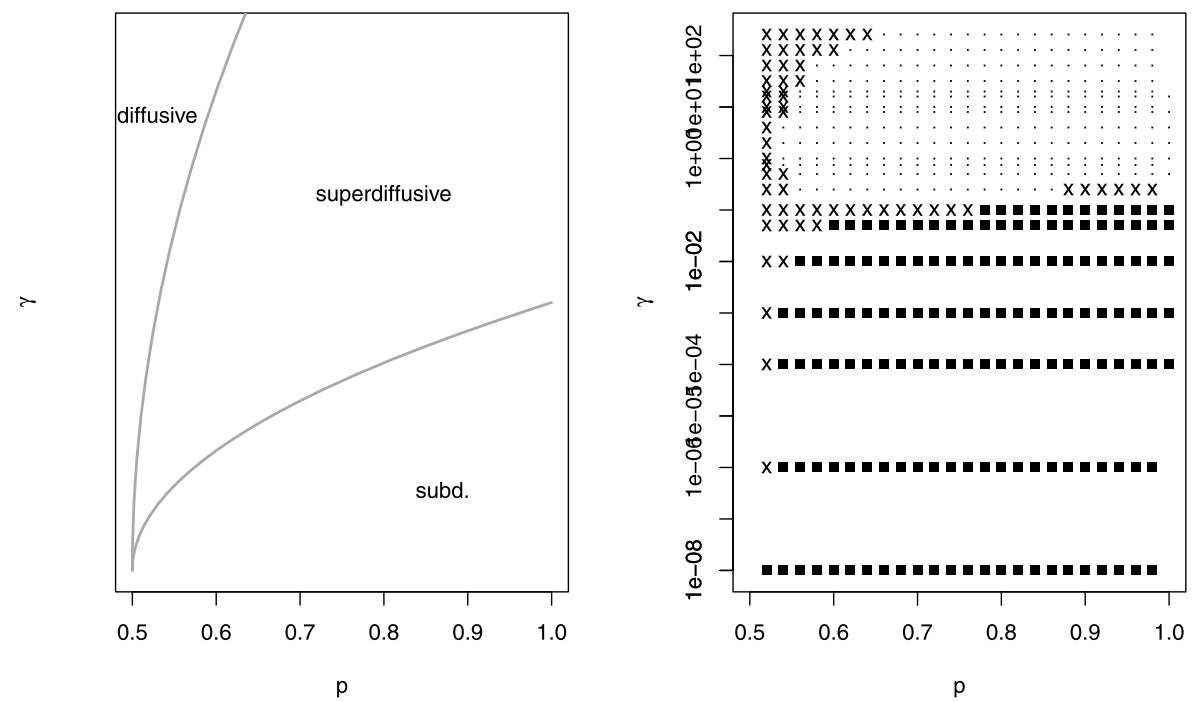

Fig. 12 The section of the phase diagram in the recurrent case, i.e. $\rho=0.5$. On the left the qualitative picture, on the right the outcome of our experiments supporting the qualitative phase diagram. The crosses, the dots, and the black squares mean that for the corresponding $(\rho, \gamma)$ points our test gave an exponent equal (diffusive), bigger (super-diffusive), or smaller (sub-diffusive) than $1 / 2$, respectively. More precisely, we assigned a cross at any estimated exponent in between $[0.49,0.51]$. Note that as in the lower part of the leaf for the static case (see Fig. 2), on the line dividing the sub-and super-diffusive regimes the scaling is diffusive 

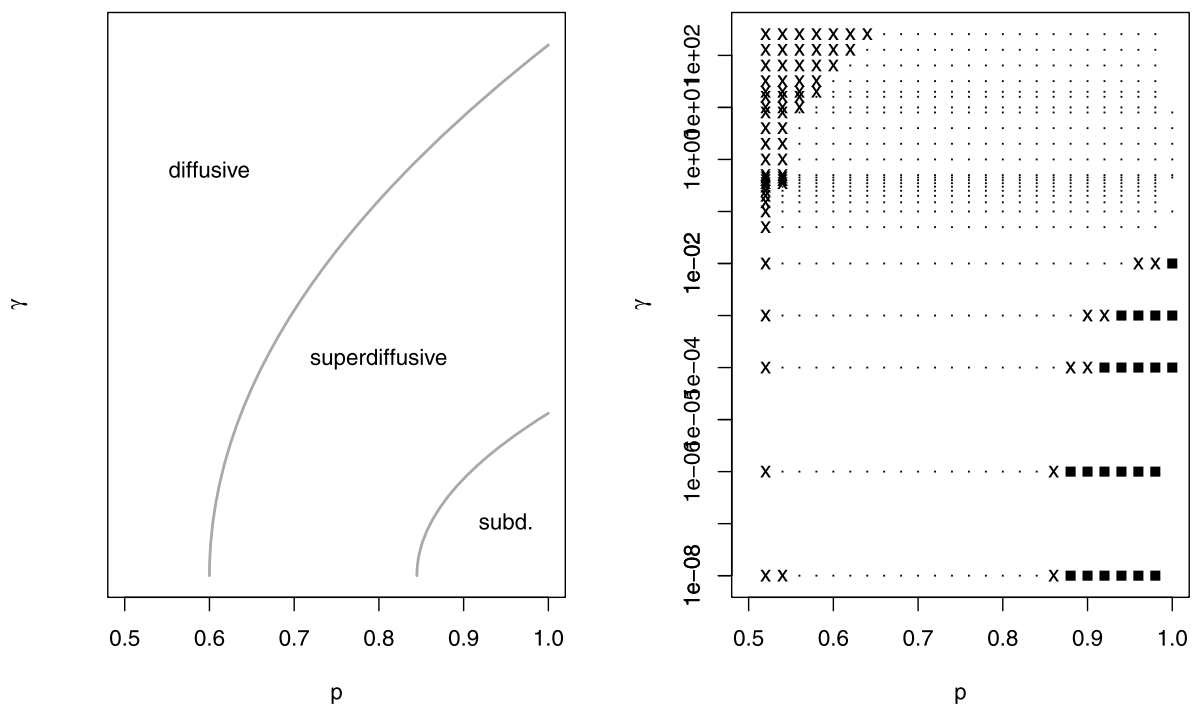

Fig. 13 The section of the phase diagram at $\rho=0.7$. On the left the qualitative picture, on the right the outcome of our experiments. As in the previous picture, crosses, dots, and squares correspond to diffusive, super- and sub-diffusive regimes, respectively
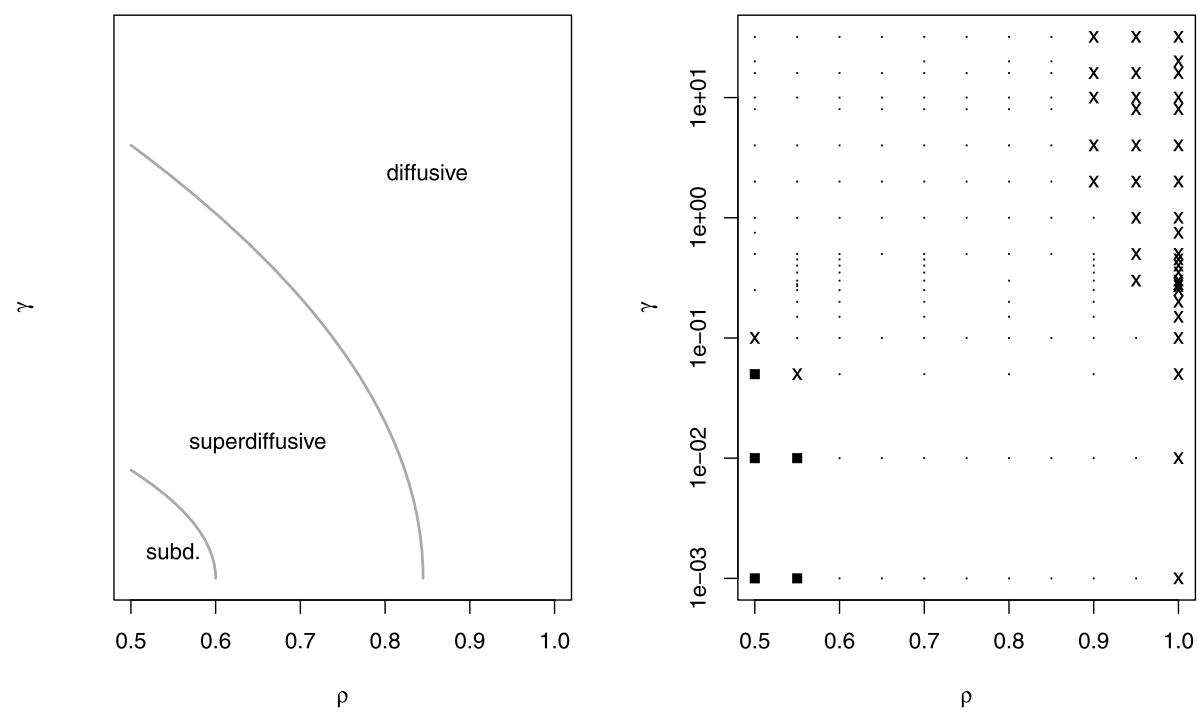

Fig. 14 The section of the phase diagram at $p=0.7$. On the left the qualitative picture, on the right the outcome of our experiments. As in the previous pictures, crosses, dots, and squares correspond to diffusive, super- and sub-diffusive regimes, respectively 


\section{p: 0.52}

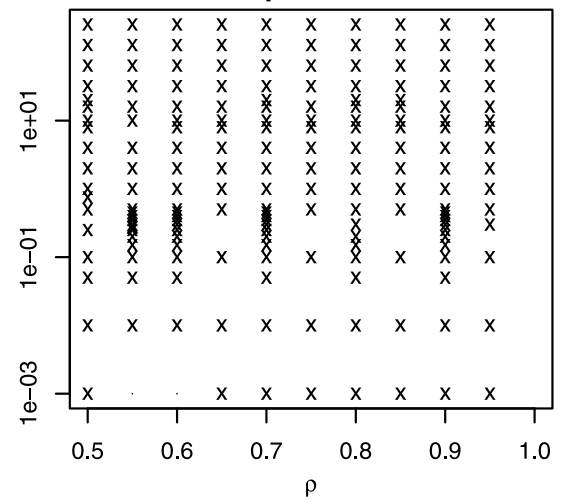

p: 0.7

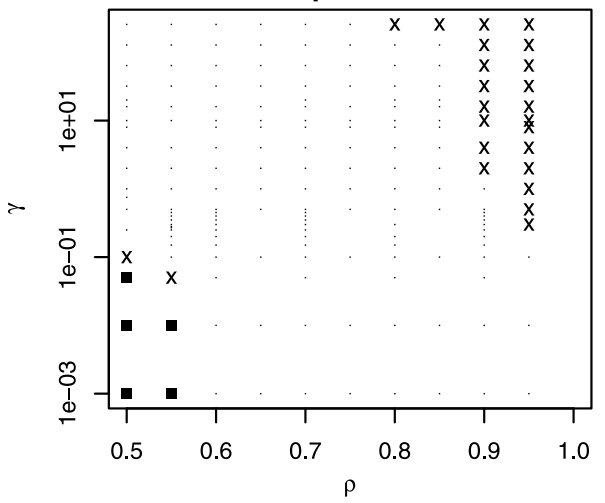

p: 0.9

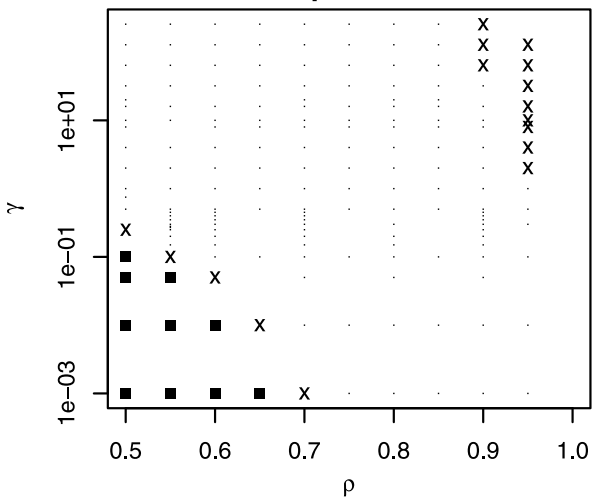

p: 0.6

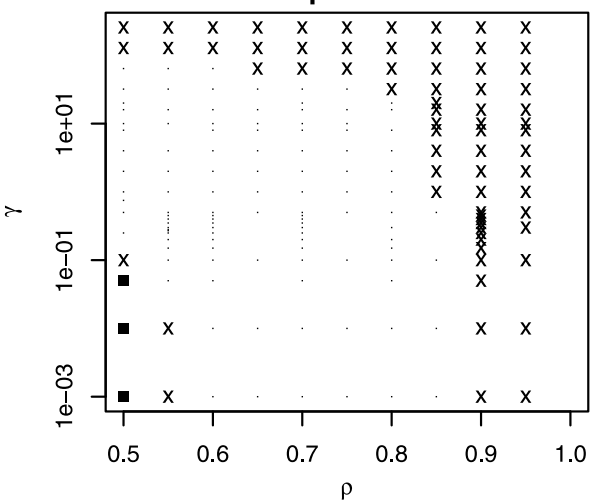

p: 0.8

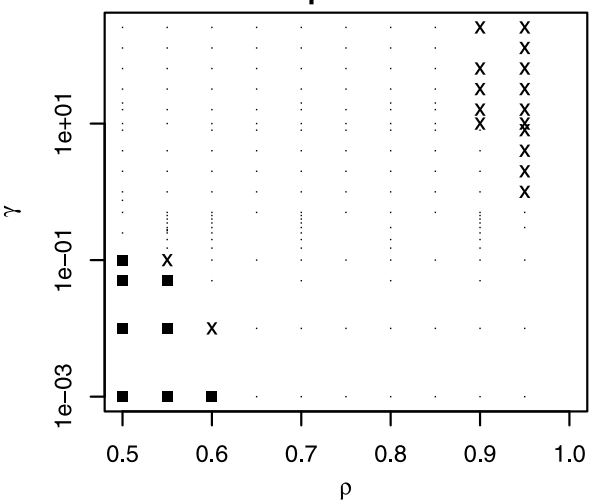

p: 0.98

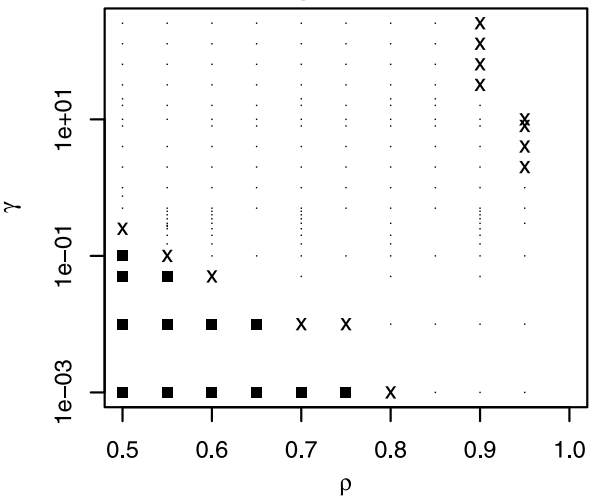

Fig. 15 Sections of data supporting the phase diagram in Conjecture 3.5 increasing in $\rho$. Crosses, dots, and squares correspond to diffusive, super- and sub-diffusive regimes, respectively 
rho: 0.5

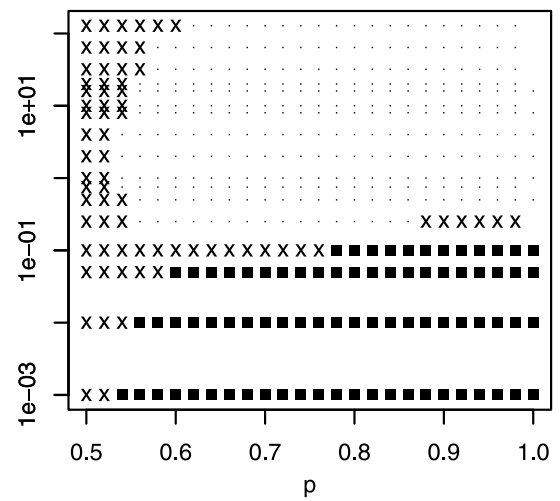

rho: 0.6

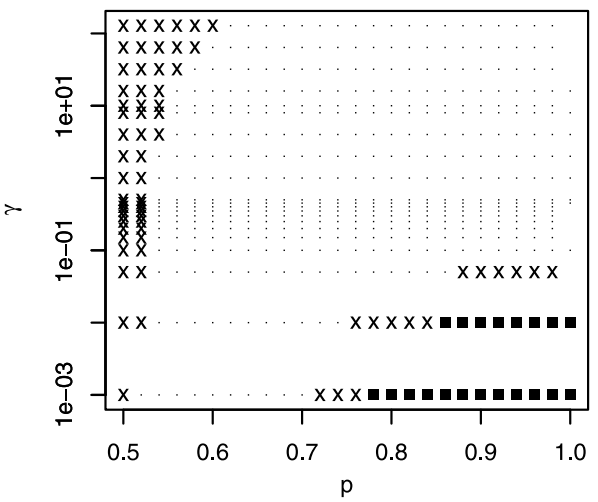

rho: 0.8

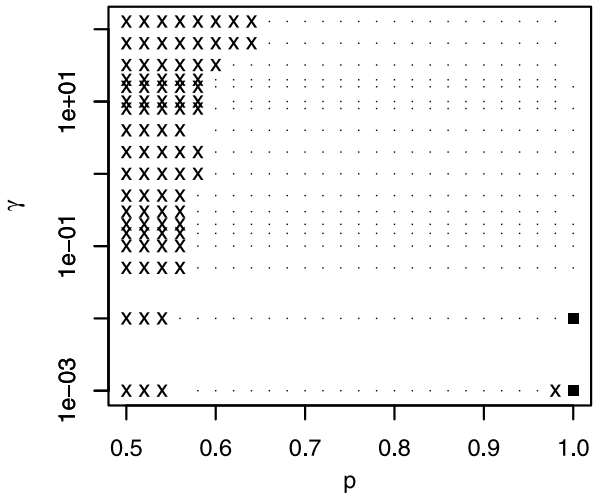

rho: 0.55

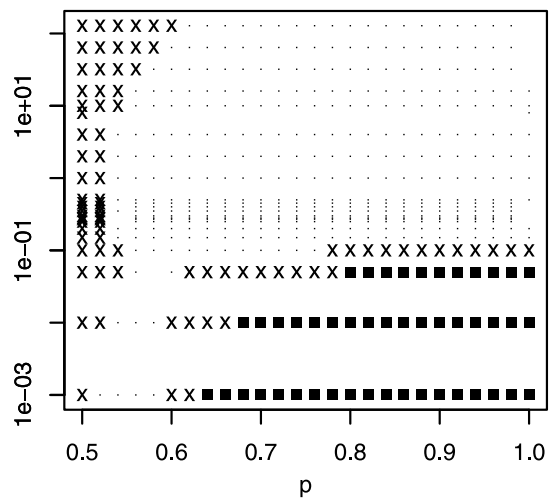

rho: 0.7

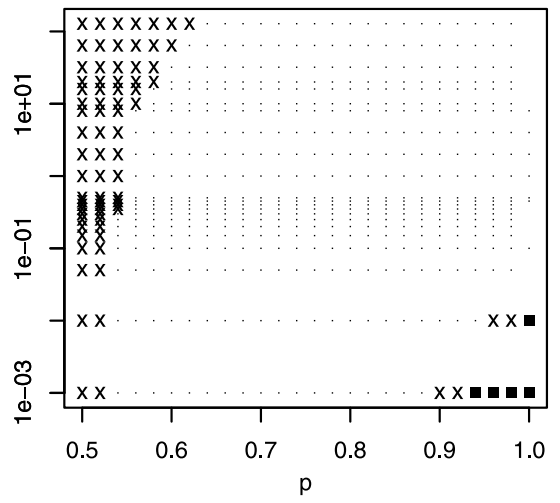

rho: 0.95

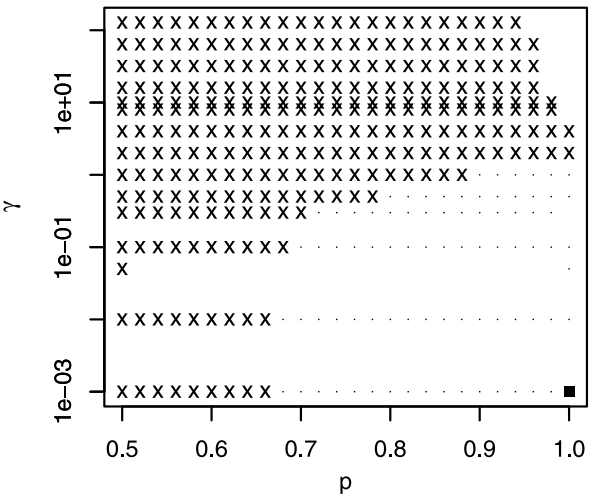

Fig. 16 Sections of data supporting the phase diagram in Conjecture 3.5 increasing in p. Crosses, dots, and squares correspond to diffusive, super- and sub-diffusive regimes, respectively 


\section{Scaling exponents in sse at rho 0.5}

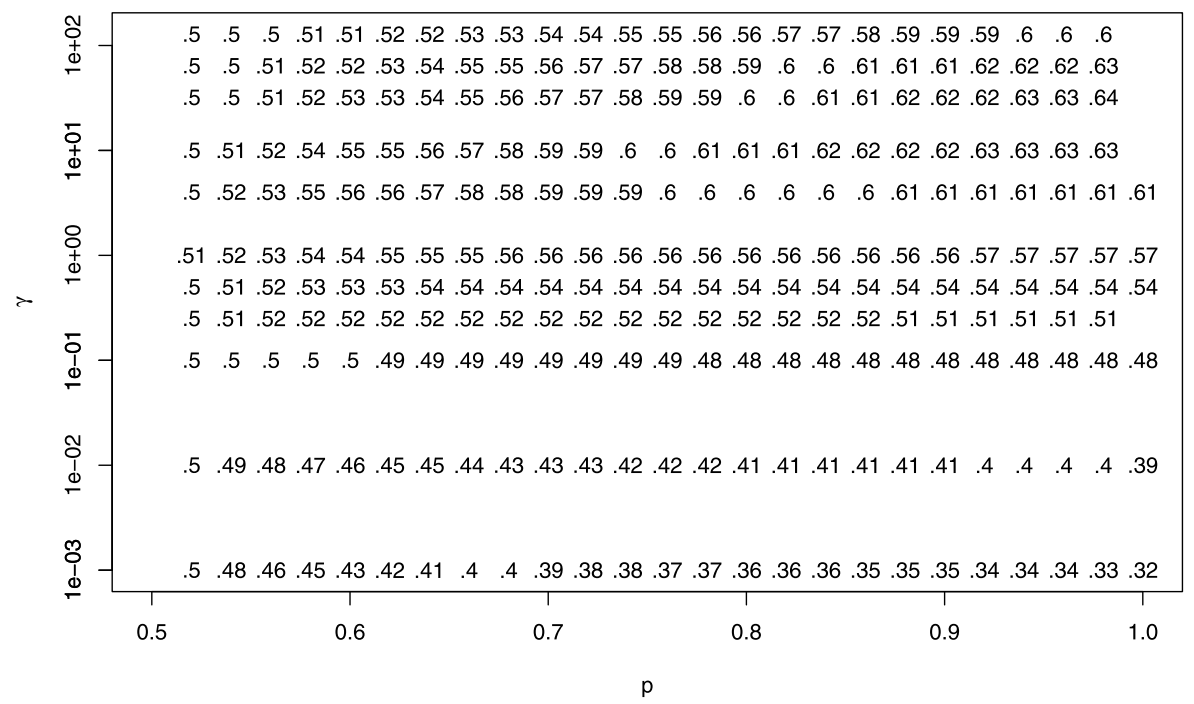

Fig. 17 A quantitative version of the phase diagram in Fig. 12. The numbers written at each point $(p, 0.5, \gamma)$ are the scaling exponents $\alpha^{\star}(p, \rho, \gamma)$ that were estimated as described below

\subsection{Scaling Exponents: Estimating the Variance for SSE}

This section is devoted to the description of the estimates we used to obtain the phase diagram described in Conjecture 3.5, i.e. the scaling exponents of $X$ in the SSE environment.

Figure 17 shows the same section as in Fig. 12 where instead of marking "diffusive and non-diffusive points", we give the explicit values of the scaling exponents.

Each of these exponents is obtained by analyzing the variances of $X$ at different times. More precisely, for each fixed triple $(p, \rho, \gamma)$, for fixed number of jumps $n$, we compute the sample standard deviation of $X_{n}(p, \rho, \gamma)$, namely,

$$
S D_{n}:=\sqrt{\frac{1}{M-1} \sum_{i=1}^{M}\left(X_{n}^{(i)}-v_{n} n\right)^{2}},
$$

over a sample of $M$ independent experiments, with $M$ of order at least $10^{3}$ (the values of $M$ are specified in the figures) and $v_{n}$ as in (3.1). We then evaluate the function

$$
\alpha(n):=\frac{\log \left(S D_{n}\right)}{\log n}
$$

on a $\log n$ scale, see Figs. 18, 19, 20, 21, 22, 23. In particular, for each value of $n$ (in the experiments $n$ grows like $2^{N}$, with $N$ specified in the figures) we have performed independent experiments. The proper scaling exponent is approximated by the value of $\alpha(n)$ with the biggest $n$ which we denote by $\alpha^{\star}=\alpha^{\star}(p, \rho, \gamma)$ (those are the numbers in Fig. 17). Indeed, assume $S D_{n}=c n^{\alpha}$, for some positive $c=c(p, \rho, \gamma)$, then $\alpha(n)=\frac{\log c}{\log n}+\alpha$ converges to $\alpha$ as $n$ goes to infinity. 

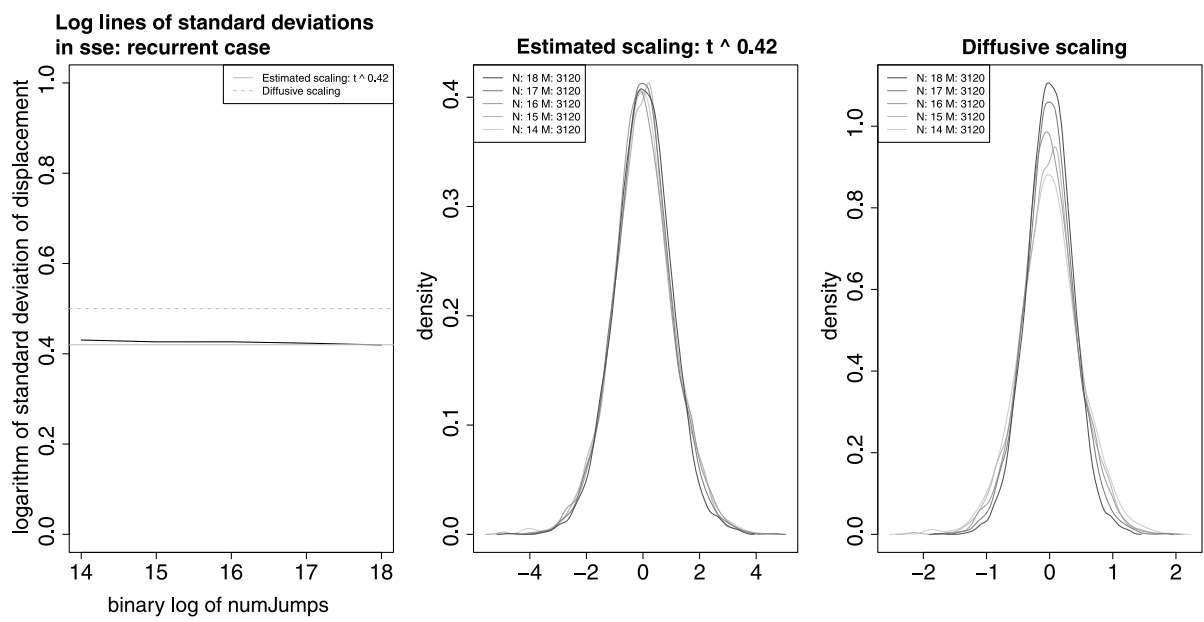

Fig. 18 Recurrent, sub-diffusive. $(p, \rho, \gamma)=(0.76,0.5,0.01)$. The left picture illustrates the behavior of the logarithm of the standard deviation of $X_{n}$ (the solid line). The dotted line represents the estimated exponent $\alpha^{\star}(0.76,0.5,0.01)$, while the dashed one represents the diffusive value 0.5 . The middle plot shows that the densities obtained at different times $n=2^{N}$ rescaled by $n^{\alpha^{\star}}$ almost perfectly match. In the right most plot, the same densities under diffusive scaling, looking carefully at the tails and around 0 , one can see that they are ordered in time and tend to concentrate in 0 as time increases. $M$ denotes the sample size. The data at different times $n$ were obtained with independent experiments

The next figures show examples of these estimates in all described regimes. Therein we plotted and overlapped the empirical densities of $X_{n}-v_{n} n$ (obtained with samples of size $M$ ) for different values of $n$ to see that indeed they coincide when rescaled by $n^{\alpha^{\star}}$. In the non-diffusive cases (i.e. $\alpha^{\star} \neq 1 / 2$ ), we also add the plot of the same empirical densities rescaled by $n^{1 / 2}$ to see that under diffusive scaling they do not coincide. In particular, they are ordered in time and the variances have the tendency of either vanishing (sub-diffusive) or concentrating (super-diffusive).

For the sake of completeness, we mention that we tried also some other methods to guess the right scaling exponent. A quite apt but not entirely stable method was to look for the exponent minimizing the total variation distance between the densities at different times. Another method was to fit an appropriate curve through the standard deviations directly. All of them gave the same qualitative picture we described here.

\section{Robustness of Simulations and More General RWRE}

\subsection{Algorithms}

To simulate the RW in the static RE, in the ISF and in the SSE, we implemented an algorithm which can be briefly described as follows.

(1) Take as INPUT $(p, \rho, \gamma, n) \in(0.5,1) \times[0.5,1) \times \mathbb{R}^{+} \times \mathbb{N}$, with $n$ being the number of jumps of the RW $X$.

(2) Consider an interval $I$ of size $3 n$ centered at the starting position of the RW.

(3) Initialize the RE according to a Bernoulli product measure of parameter $\rho$.

(4) At exponential time of rate 1, update the RW position according to the underlying state of the RE. 

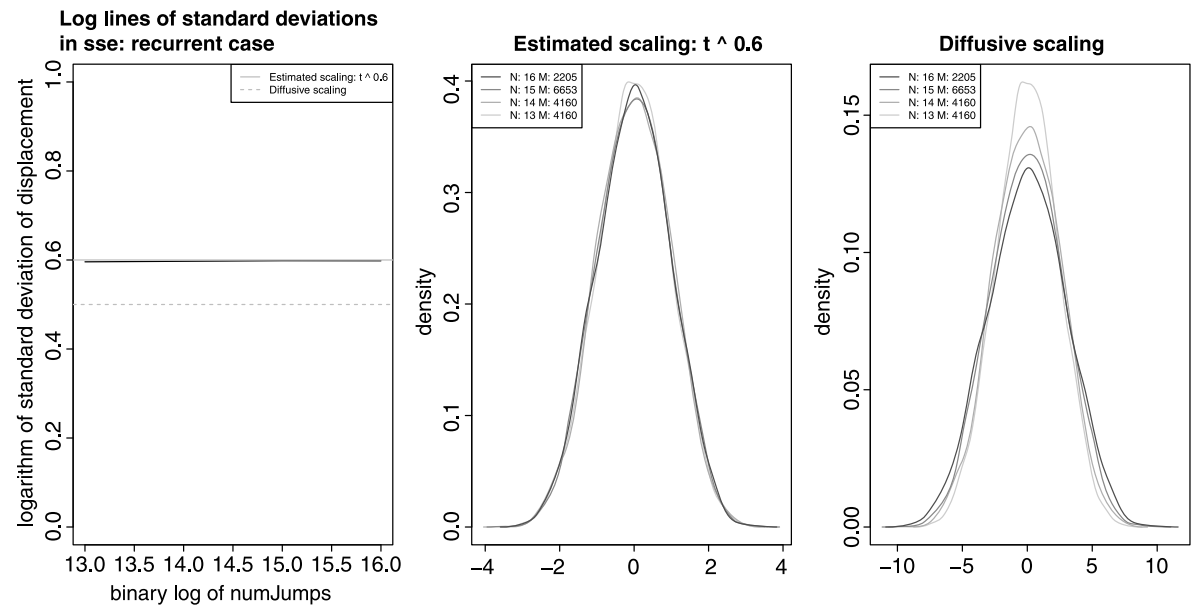

Fig. 19 Recurrent, super-diffusive. $(p, \rho, \gamma)=(0.8,0.5,4)$. As in the previous figure, the left picture illustrates the behavior of the logarithm of the standard deviation of $X_{n}$ (the solid line). The dotted line represents the estimated exponent $\alpha^{\star}(0.8,0.5,4)$, while the dashed one represents the diffusive value 0.5 . The middle plot shows that the densities obtained at different times $n=2^{N}$ rescaled by $n^{\alpha^{\star}}$ almost perfectly match. In the right most plot, the same densities under diffusive scaling, note that in this case they are ordered in time and tend to vanish as time increases. $M$ denotes the sample size. The data at different times $n$ were obtained with independent experiments
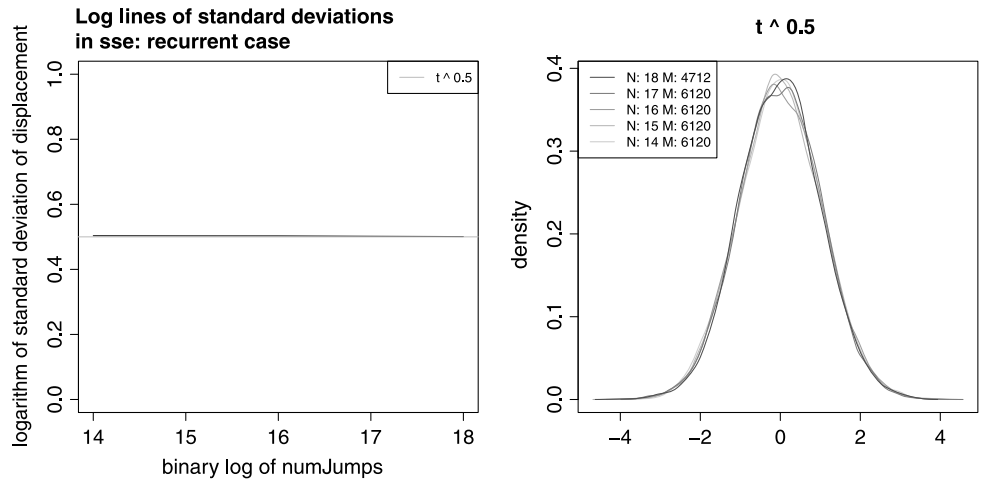

Fig. 20 Recurrent, diffusive. $(p, \rho, \gamma)=(0.56,0.5,0.1)$. As in the previous figures, the left picture illustrates the behavior of the logarithm of the standard deviation of $X_{n}$. The dotted line represents the estimated exponent $\alpha^{\star}(0.6,0.5,0.1)$. The right plot shows that the densities obtained at different times $n=2^{N}$ coincide under diffusive rescaling

(5) For each $x$ in $I$, at exponential time of rate $\gamma$ update the RE at position $x$.

(6) Give as OUTPUT the position of the RW after $n$ steps.

Note that Step (5) depends on the RE. For the static case, $\gamma=0$ implies that we never update the RE. In the ISF case, due to the independence in space of the dynamics, we update the RE only locally at the position of the RW $X$ (see Fig. 6). The case of the SSE has been implemented using a version of the SSE either on the torus (i.e. with periodic boundary conditions) or by sampling at rate $\gamma$ the state of the RE at the boundaries of $I$ from $v_{\rho}$ (both approaches produce the same outcome). In particular, the size of $I$ has been chosen of size 

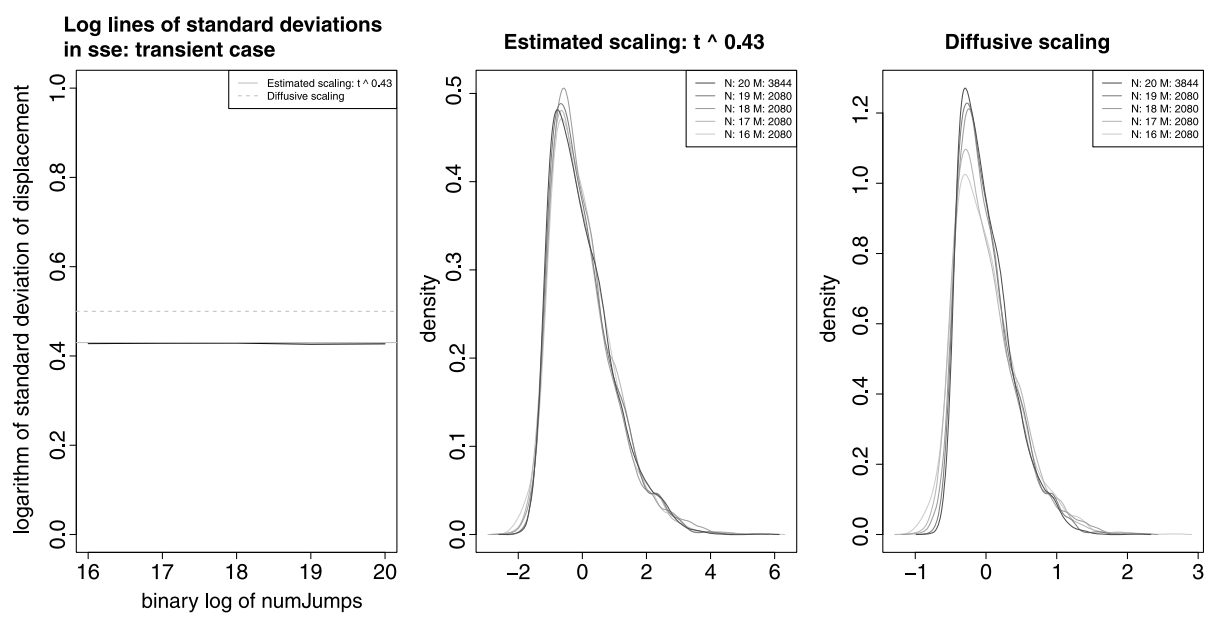

Fig. 21 Transient, sub-diffusive. $(p, \rho, \gamma)=(0.74,0.55,0.001)$. Estimated reliable time: $\bar{n}=2^{38}$ (see Sect. 4.2)
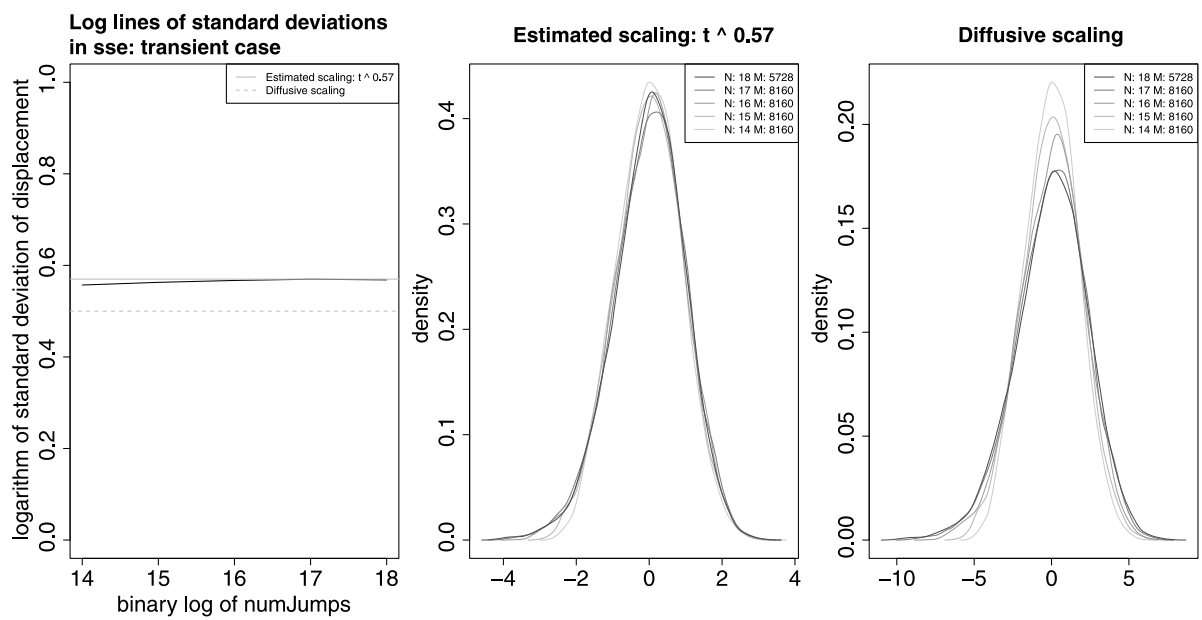

Fig. 22 Transient, super-diffusive. $(p, \rho, \gamma)=(0.86,0.6,0.25)$. Estimated reliable time: $\bar{n}=2^{12}$, effective running time $n=2^{18}$ (see Sect. 4.2)

$3 n$. Particles coming from the boundaries will typically travel a distance of order $\sqrt{\gamma n}$ up to time $n$ which guarantees that the dynamics of $X$ is not significantly affected by those particles (note that the biggest $\gamma$ we considered in our numerics is of order 100).

\subsection{Heuristics Versus Locality}

Conjecture 3.5 suggests a completely different scenario for the scaling limits depending on whether the RE is fast (ISF) or slowly mixing (SSE). This latter result is certainly the most interesting suggested by our numerics. Indeed, in all the papers dealing with RWDRE only diffusivity has been proven since, due to technical difficulties, most of the tools available are 

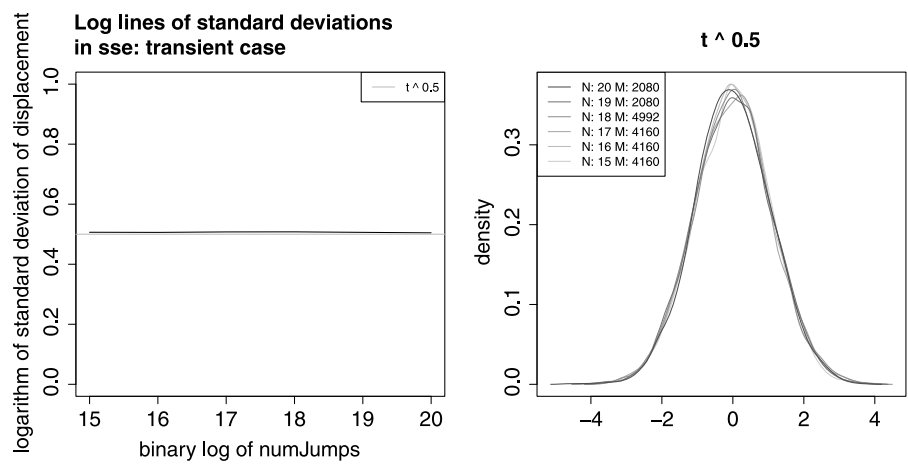

Fig. 23 Transient, diffusive. $(p, \rho, \gamma)=(0.56,0.9,0.001)$. Estimated reliable time: $\bar{n}=2^{305}$ (see Sect. 4.2)

not suitable to treat RE presenting space-time correlations except under strong mixing conditions. The non-diffusivity in the experiments for the SSE could still be a local phenomenon. Namely, the time we run the algorithm could in principle be too small and what we are observing is only a perturbation of the static case or due to other local effects: e.g. presence of constants, too small $\gamma$, parameters in a neighborhood of the boundary of two phases or close to degenerate cases. Therefore, to be more confident that we are truly observing the right asymptotic scalings, and to have some stronger evidence in favor of Conjecture 3.5, we need to understand whether the running time of each experiment is large enough.

We present here a heuristic argument to guess a reliable running time for small $\gamma$. The idea is that in each simulation the RW should perform a number of jumps sufficiently large to ensure that the time it takes the walker to cross a typical trap is comparable with the time in which this trap dissolves.

Consider a trap of size $L$ of consecutive holes (a stretch of size $L$ in $\mathbb{Z}$ where the state of the RE is 0 ), assume the walker starts at time 0 on the left-most hole. Let $\tau_{L}$ denote the number of jumps the walker needs before reaching the right-most hole of the trap. By using a standard gambler's ruin argument we see that

$$
\mathbb{E}\left[\tau_{L}\right] \geq \frac{(p / q)^{L}-1}{(p / q)-1} .
$$

On the other hand we can estimate the "mixing time" of the trap to be of order $\left(\gamma^{-1} L\right)^{2}$. Indeed, a particle at the boundary of the trap (since it is performing a simple symmetric RW at rate $\gamma$ ) would need in average this amount of time to cross the trap.

If $\mathbb{E}\left[\tau_{L}\right]$ is much smaller than $\left(\gamma^{-1} L\right)^{2}$, this would mean that within the time the RW crosses the trap, the dissolvence effect due to the dynamics of the SSE is not big enough to play a substantial role. Therefore we would like to choose $L$ big enough so that the two quantities are at least comparable, namely, we have to solve (when possible) the equation

$$
\frac{(p / q)^{L}-1}{(p / q)-1}=\left(\gamma^{-1} L\right)^{2} .
$$

Once we compute (numerically) the solution $L=L(p, \gamma)$ of this equation, we have to be sure that the RW $X$ travels a distance big enough so that it will meet with high probability such a big trap. The probability of observing such a disaster can be easily estimated by a geometric argument since the probability of a stretch of hole of size $L$ is $(1-\rho)^{L}$. Therefore to be sure that the RW $X(p, \rho, \gamma)$ will meet and cross at least one disaster with high 
probability we have to run the algorithm for $\bar{n}=\bar{n}(p, \rho, \gamma)$ steps, with $\bar{n}$ big enough so that $X_{\bar{n}} \geq L(1-\rho)^{L}$ with high probability.

It turns out that the order of such a $\bar{n}(p, \rho, \gamma)$ varies a lot depending on $(p, \rho, \gamma)$. In most of the parameter space is unfortunately too big to be achieved in a reasonable amount of computing time. In the caption of Figs. 21, 22, 23 we wrote the explicit corresponding values of $\bar{n}$. The fact that in most of the cases we could not run the algorithm up to such an $\bar{n}$, does not imply that the outcome of the associated experiment is only local and not asymptotically reliable, as the argument to deduce $\bar{n}$ is only a rough estimate. On the other hand, the fact that for several super-diffusive cases (as in the example in Fig. 22) we could achieve the associated $\bar{n}$ is a strong suggestion in favor of Conjecture 3.5.

\subsection{Concluding Remarks}

As mentioned before, at the current state of the art, most of the tools (regeneration, renormalization, coupling, martingale approximation, etc.) developed to analyze RWRE (both static and dynamic REs) are still inappropriate to deal with space-time correlations unless the environment satisfies some uniform and fast enough mixing condition. This is not the case in the example of the SSE, that is why the LLN and the scaling limits for a RW driven by these types of REs are serious mathematical challenges. It is reasonable to think that if the observed unusual regimes in Conjecture 3.5 will be rigorously proven, a similar phenomenology can occur for other one dimensional "slowly mixing" RE (e.g. other conservative particle systems) providing that the RW is nearest-neighbor and has local drifts in both directions. In principle, we might expect some analogous scenario even in dimensions higher than one for static and dynamic REs presenting again some strong correlation structure. The latter observation is supported by the fact that a one-dimensional RW in a dynamic $\mathrm{RE}$ can be seen as a two dimensional directed RW in a static RE by interpreting the time as an extra spatial dimension (see e.g. [3]).

We conclude with a table summarizing briefly some of the one-dimensional rigorous and numerical results we presented so far. Cells in gray mean that the corresponding result is non-rigorous.

\begin{tabular}{llll}
\hline & Static & Dynamic & \\
\cline { 3 - 4 } & & SSE (slowly mixing) & ISF (fast mixing) \\
\hline Recurrence & $\rho=1 / 2$ & $\rho=1 / 2$ & $\rho=1 / 2$ \\
LLN with $\rho \neq 1 / 2$ & $v \geq 0$ & $\begin{array}{l}v>0, \\
\text { unclear for small } \gamma\end{array}$ & $v>0$ \\
Scaling & anomalous & $\begin{array}{l}\gamma \text { big: diffusive, } \gamma \\
\text { small: anomalous }\end{array}$ & diffusive \\
LDP rate $t$ for $v>0$ & flat piece & flat piece & unique zero \\
\hline
\end{tabular}

Acknowledgements We would like to thank Frank den Hollander, Renato dos Santos, Vladas Sidoravicius and Florian Völlering for fruitful discussions during the preparation of this paper. The simulations were performed on the Schrödinger cluster of the University of Zürich, where we gratefully acknowledge the support of C. Bolliger, A. Godknecht, and R. Graf, and at the Institute of Mathematics, thanks to R. Ostertag and C. Rose. 


\section{References}

1. Avena, L.: Random walks in dynamic random environments. PhD thesis, Leiden (2010). Available on the online catalogue of the Leiden University Library: www.catalogus.leidenuniv.nl,

2. Avena, L., den Hollander, F., Redig, F.: Large deviation principle for one-dimensional random walk in dynamic random environment: attractive spin-flips and simple symmetric exclusion. Markov Process. Relat. Fields 16, 139-168 (2010)

3. Avena, L., den Hollander, F., Redig, F.: Law of large numbers for a class of random walks in dynamic random environments. Electron. J. Probab. 16, 587-617 (2011)

4. Avena, L., dos Santos, R., Völlering, F.: Transient random walk in symmetric exclusion: limit laws and an Einstein relation. Preprint (2011). Available at arXiv:1102.1075

5. Bandyopadhyay, A., Zeitouni, O.: Random walk in dynamic Markovian random environment. ALEA Lat. Am. J. Probab. Math. Stat. 1, 205-224 (2006)

6. Bogachev, L.V.: Random walks in random environments. In: Encyclopedia of Mathematical Physics, vol. 4, pp. 353-371. Elsevier, Oxford (2006)

7. Boldrighini, C., Minlos, R.A., Pellegrinotti, A.: Random walk in a fluctuating random environment with Markov evolution. In: On Dobrushin's Way. From Probability Theory to Statistical Physics. Am. Math. Soc. Transl., vol. 198, pp. 13-35 (2000)

8. Boldrighini, C., Minlos, R.A., Pellegrinotti, A.: Random walks in quenched i.i.d. space-time random environment are always a.s. diffusive. Probab. Theory Relat. Fields 129, 133-156 (2004)

9. Boldrighini, C., Ignatyuk, I.A., Malyshev, V., Pellegrinotti, A.: Random walk in dynamic environment with mutual influence. Stoch. Process. Appl. 41, 157-177 (1992)

10. Bricmont, J., Kupiainen, A.: Random walks in space time mixing environments. J. Stat. Phys. 134, 9791004 (2009)

11. Comets, F., Gantert, N., Zeitouni, O.: Quenched, annealed and functional large deviations for onedimensional random walk in random environment. Probab. Theory Relat. Fields 118, 65-114 (2000)

12. den Hollander, F., Kesten, H., Sidoravicius, V.: Random walk in a high density dynamic random environment. Preprint (2012)

13. den Hollander, F., dos Santos, R., Sidoravicius, V.: Law of large numbers for non-elliptic random walks in dynamic random environments. Preprint (2011). Available at arXiv:1103.2805

14. Dolgopyat, D., Keller, G., Liverani, C.: Random walk in Markovian environment. Ann. Probab. 36, 1676-1710 (2008)

15. Greven, A., den Hollander, F.: Large deviations for a random walk in random environment. Ann. Probab. 22, 1381-1428 (1994)

16. Kesten, H.: The limit distribution of Sinai's random walk in random environment. Physica 138, 299-309 (1986)

17. Kesten, H., Kozlov, M.V., Spitzer, F.: A limit law for random walk in a random environment. Compos. Math. 30, 145-168 (1975)

18. Liggett, T.M.: Interacting Particle Systems. Grundlehren der Mathematischen Wissenschaften, vol. 276. Springer, New York (1985)

19. Rassoul-Agha, F., Seppalainen, T.: An almost sure invariance principle for random walks in a space-time random environment. Probab. Theory Relat. Fields 133, 299-314 (2005)

20. Redig, F., Völlering, F.: Limit theorems for random walks in dynamic random environment. Preprint (2011). Available at arXiv:1106.4181

21. Sinai, Ya.G.: The limiting behavior of one-dimensional random walk in random environment. Theory Probab. Appl. 27, 256-268 (1982)

22. Solomon, F.: Random walks in a random environment. Ann. Probab. 3, 1-31 (1975)

23. Sznitman, A.S.: Lectures on random motions in random media. In: Ten Lectures on Random Media. DMV-Lectures, vol. 32. Birkhäuser, Basel (2002)

24. Varadhan, S.R.S.: Large deviations for random walks in a random environment. Commun. Pure Appl. Math. 56, 309-318 (2003)

25. Zeitouni, O.: Random walks in random environment. In: XXXI Summer School in Probability, SaintFlour, 2001. Lecture Notes in Math., vol. 1837, pp. 189-312 (2004)

26. Zeitouni, O.: Random walks in random environments. J. Phys. A, Math. Gen. 39, R433-R464 (2006) 